\title{
Human well-being impacts of terrestrial protected areas
}

Andrew S Pullin ${ }^{1 *}$, Mukdarut Bangpan ${ }^{2}$, Sarah Dalrymple ${ }^{1}$, Kelly Dickson ${ }^{2}$, Neal R Haddaway ${ }^{1}$, John R Healey ${ }^{3}$, Hanan Hauari ${ }^{2}$, Neal Hockley ${ }^{3}$, Julia P G Jones ${ }^{3}$, Teri Knight ${ }^{1}$, Carol Vigurs ${ }^{2}$ and Sandy Oliver ${ }^{2}$

\begin{abstract}
Background: Establishing Protected Areas (PAs) is among the most common conservation interventions. Protecting areas from the threats posed by human activity will by definition inhibit some human actions. However, adverse impacts could be balanced by maintaining ecosystem services or introducing new livelihood options. Consequently there is an ongoing debate on whether the net impact of PAs on human well-being at local or regional scales is positive or negative. We report here on a systematic review of evidence for impacts on human well-being arising from the establishment and maintenance of terrestrial PAs.

Methods: Following an a priori protocol, systematic searches were conducted for evidence of impacts of PAs post 1992. After article title screening, the review was divided into two separate processes; a qualitative synthesis of explanations and meaning of impact and a review of quantitative evidence of impact. Abstracts and full texts were assessed using inclusion criteria and conceptual models of potential impacts. Relevant studies were critically appraised and data extracted and sorted according to type of impact reported. No quantitative synthesis was possible with the evidence available. Two narrative syntheses were produced and their outputs compared in a metasynthesis.
\end{abstract}

Results: The qualitative evidence review mapped 306 articles and synthesised 34 that were scored as high quality. The quantitative evidence review critically appraised 79 studies and included 14 of low/medium susceptibility to bias. The meta-synthesis reveals that a range of factors can lead to reports of positive and negative impacts of PA establishment, and therefore might enable hypothesis generation regarding cause and effect relationships, but resulting hypotheses cannot be tested with the current available evidence.

Conclusions: The evidence base provides a range of possible pathways of impact, both positive and negative, of PAs on human well-being but provides very little support for decision making on how to maximise positive impacts. The nature of the research reported to date forms a diverse and fragmented body of evidence unsuitable for the purpose of informing policy formation on how to achieve win-win outcomes for biodiversity and human well-being. To better assess the impacts of PAs on human well-being we make recommendations for improving research study design and reporting.

Keywords: National Park, Reserve, Community, Governance, Conservation, Poverty, Development, Biodiversity, Systematic review

\footnotetext{
* Correspondence: a.s.pullin@bangor.ac.uk

${ }^{1}$ Centre for Evidence-Based Conservation, School of Environment, Natural

Resources and Geography, Bangor University, LL57 2UW Bangor, Gwynedd, UK

Full list of author information is available at the end of the article
} 


\section{Background}

The concept and practice of protecting areas for the purposes of conservation has been at the heart of conservation policy since its inception in the 19th Century. The idea that intervening to protect areas from human activity is an effective way of conserving species and habitats and preventing habitat loss and species extinction is arguably as pervasive today as it was when the first protected areas (PAs) were established (MEA 2005). The central place of PAs in the conservation movement has been reflected in the increase in both the number of PAs and the area of land and sea placed under protection. The proportion of total area of land under some form of protection has now reached nearly $13 \%[1,2]$.

The process of protecting areas from the threats posed by human activity will by definition inhibit some human actions and therefore has the potential to negatively impact human well-being. There are many historical records to suggest that few PAs were uninhabited wildernesses before designation, and the early history of PAs, for example in the USA and East Africa, is of forced eviction and persecution of local communities by colonial powers [3]. This scenario has continued in some countries with, in some cases, the colonial powers being replaced by multinational corporations or international conservation Non-Governmental Organisations (NGOs) [4]. The problem of negative impacts of PAs on human well-being gained official recognition in the Convention on Biological Diversity (CBD) arising from the Rio Summit in 1992. The principle that PAs should do no harm to local people was only established at the World's Park Congress in 2003 (at which, the Durban Accord was proclaimed). Prior to 1992, the establishment plans of PAs did not normally have objectives concerning human well-being.

However, negative impacts could be balanced by positive impacts as PAs may also improve human well-being and alleviate poverty [5]. By preventing conversion of natural habitats, PAs may improve the provision of some valued ecosystem services to some users. For example, downstream farmers may benefit from conservation of forested watersheds [6]. PAs may also directly introduce new livelihood options into a region through the expansion of tourism or research, or improvements to infrastructure may indirectly result in economic development.

Recently there has been considerable debate on whether, apart from their effects on global environmental benefits, the net impact of PAs on human well-being at local or regional scales are positive or negative [7-10]. There is concern that continuing with a policy of PA establishment could conflict with goals of poverty alleviation [11]. The CBD Aichi targets include a target of $17 \%$ of terrestrial and inland areas covered by well-managed PAs by 2020 . Future policy decisions on support for PA establishment and future management need to be informed by the best available evidence on their human well-being impacts. That there have been and continue to be, some major negative impacts on local communities caused by the establishment of some protected areas, is not in dispute. However, of particular interest to policy makers is the balance of positive and negative impacts on human livelihoods that arise from the PA establishment, the distribution of these benefits and costs, and the factors that might cause this to vary.

We report here on the conduct and outcome of a systematic review of evidence for impacts on human wellbeing arising from the establishment and maintenance of terrestrial PAs. Establishing the state of the evidence base through systematic review will inform decision making concerning future investment in PAs and future research needs. The review question was formulated by the Scientific and Technical Advisory Panel (STAP) of the Global Environment Facility (GEF).

At the outset of the review the following broad categories were posed by STAP as a starting point for a conceptual model concerning the potential impact of PA establishment (or change in PA status) on people and/or the communities of which they are part. These categories and associated questions were used to guide development of specific evidence inclusion criteria (categories were subsequently modified based on an in-depth understanding of the literature in order to code and present the available evidence, see methods).

\section{Livelihood strategies}

Did the establishment or change in status of the PA or management activities within the PA generate or decrease specific production opportunities (e.g. more demand for labour, herding activities and associated products no longer viable, new demand for particular food, handicraft, services or products etc.)? Did the PA influence (i.e. increase or decrease) migration generally, and of particular social groups? Has this differentially impacted (positively or negatively) the most vulnerable groups in local communities (e.g. women, children, poorest sectors of the community)?

\section{Social capital}

Did the establishment and management of the PA affect the development of social networks? Did it positively or negatively impact education and capacity building, e.g. by generating or decreasing opportunities for formal and/ or informal education? Has PA establishment differentially affected more vulnerable groups (e.g. women, children, poorest sectors within local communities) in a positive or negative way?

\section{Empowerment}

Did the PA empower or disempower local communities and any particular social groups? Were new organizations/ 
institutional arrangements that represent the interests of communities and any particular social groups created or existing ones undermined? Have these organizations developed activities aimed at improving their livelihoods (e.g., legislation to support local livelihoods, land tenure, co-management of local resources, other social benefits) or have existing activities been negatively affected?

\section{Human rights}

Whilst recognising that the scope of human rights is very broad, in this review we focus on the following question; were the rights of any local stakeholders, affected either positively or negatively by the PA (considering, e.g., rights to education, adequate access to food, clothing, health, choices)?

\section{Access to ecosystem goods and services and natural resources essential for well-being}

Did the PA have any positive or negative impact on access to ecosystem services and natural resources? For example were there changes in the cost (in terms of money, level of effort, or time) in obtaining firewood, clean water, and other resources/services? Was access to culturally significant places (e.g. sacred grounds) affected? Did self-sufficiency in food (by locally cultivating, hunting, raising animals or gathering) or access to medicinal plants change? Has this been a consequence of the direct impact of the PA through legal prohibition of access or indirect as a consequence of changes in infrastructure and/or institutions? Have any of these positive or negative impacts been disproportionably high or low on particular sectors of society?

Measuring impacts needs to take into account that terrestrial PAs are varied in their status, management and objectives. For example, many protected areas will have been established with a primary aim of landscape or biodiversity conservation, possibly at a time when potential impacts on local human well-being were not widely considered. Types of PAs have been categorised by the World Commission on Protected Areas (WCPA) of the International Union for the Conservation of Nature (IUCN) and are used to classify entries in the World Database of Protected Areas (http://www.protectedplanet.net/). The PA categories reflect the range of management objectives of different PAs, from strict protection of nature or wilderness, conservation of ecosystems, protection of national monuments or management of important habitat or species (categories I-IV) through to objectives which place human use of the landscape much more centrally (categories V-VI). Progressively, since 1992 the requirement of PAs to 'Deliver benefits to resident and local communities consistent with the other objectives of management' has become a common objective [12] with the categorisation representing a gradation of increasing human intervention.
In this review we also recognise that the impacts of PAs on human well-being are likely to be highly context dependent and vary over the lifetime of the PA. For example, initial establishment may have significant and immediate negative impacts on the communities within the PA but in the longer term there may be positive impacts on well-being. Similarly, spatial context of the PA and communities within and around it will influence impact. Some communities will be located in a PA, some in buffer zones around the edge and others more distant. Other PAs may be close by and exert an influence. Presence or absence of infrastructure may influence the spatial scale over which impacts occur. Impacts that are negative 'locally' may have to be balanced against impacts that are positive but experienced more widely or more remotely.

Given the diversity of PAs, contexts of establishment and of communities affected, this review did not aim to estimate a mean effect of PA establishment on local communities but to critically appraise the range of effects that have been reported. The nature and scope of the question posed in this review (including as it does issues of empowerment and social capital) suggested to the Stakeholders and the Review Team that a combination of quantitative and qualitative evidence might provide the most reliable base with which to inform future decision making.

\section{Objective of the review}

The primary research question was:

What are the human well-being impacts of terrestrial protected areas?

We aimed to synthesise the empirical evidence of positive, negative or neutral impacts of PAs on human wellbeing at the local to regional scales, with emphasis on local communities and contemplating as broad a definition of well-being as possible (see categories to be considered below).

We were also interested in two secondary questions;

1. How are costs and benefits distributed among and within local communities living inside and in the buffer zones of PAs (by socio-economic status, gender, age etc.)? 2 . How do costs and benefits vary with governance, resource tenure arrangements, and site characteristics? As the aim is to assess the human well-being impacts of PAs in their current form and capture lessons for future interventions, relevant evidence may be provided by studies that generate hypotheses about impact, as well as studies that test hypotheses about impact.

\section{Methods}

Design of review

An a priori protocol was established, peer reviewed and posted on the CEE website by CEE [13]. This review was 
divided into two separate processes; a qualitative synthesis of people's views, observations and related documentary evidence (led by the EPPI-Centre) and a synthesis of quantitative evidence of impact, including people's attitudes and views (led by Bangor University). Henceforth these will be referred to as 'qualitative' and 'quantitative' as reflected in the protocol and the methods below. A single systematic search was conducted to identify potentially relevant studies to answer the review questions. Initial screening of title was undertaken by Bangor University. Further supplementary searching, screening, coding and synthesis were conducted independently by each of the review teams. Thus, the searching phase and initial screening by title were not separate, but thereafter the report sections reflect the different approaches.

\section{Searches}

Search terms were selected to capture all information pertaining to PAs (the intervention) and relevant outcomes associated with human well-being. No search terms for the study population, in this case local communities, were used as these were not likely to be included in the title or abstract and therefore their incorporation in the search might have risked excluding relevant studies. The use of the asterisk denotes a wildcard character that prevents plurals or alternative word endings being excluded. The search terms are as follows:

Exposure: protected area*, nature reserve*, wilderness area*, national park*, natural monument*, natural feature*, management area*, world heritage site*, biosphere reserve*, biodiversity conservation.

Outcomes: poverty, human well*, socio-econom*, econom*, human health, livelihood*, social capital, social welfare, empowerment, equity, ecosystem service* $^{*}$, perception*, attitude*.

Where the databases searched could accommodate all search terms simultaneously, they were separated using Boolean operators. The search terms are grouped by intervention and outcome and we refer to these groups as 'sets'. The Boolean operator 'OR' was used to separate search terms within a set and the operator 'AND' used to separate the two sets thus ensuring that the search returned references including at least one term from each set.

In many cases, the literature databases could not accommodate all the search terms above and therefore search strings had to be adapted as necessary. In some cases, this meant using pairs of search terms, one from each set, separated by 'AND'. Occasionally, search terms had to be entered individually and/or the database did not accommodate Boolean operators, in these cases only the search terms pertaining to the intervention were used. Full details of the search are recorded in Additional file 1.
Where studies are reported in other languages, relevance was assessed initially from their titles and abstracts (translated if necessary). Non-English language articles that could not be considered in this review were recorded for future assessment (see Additional file 2).

For the qualitative synthesis, non-English papers were translated using Google Translate online translation tool. Google Translate was tested for accuracy using a sample report that included both and English and non-English abstracts. By translating the non-English abstract of the paper and comparing with the English abstract given in the same paper we were able to see how well the tool 'understood' and conveyed the same meanings in the text. Google Translate was found to be a fairly accurate tool, although more accurate on some languages than others. Using Google Translate, non-English articles were translated then screened on full text and included or excluded.

\section{Estimating the comprehensiveness of the database search}

All search terms were included in a search string formatted according to requirements for searching in the Web of Knowledge database. A set of 18 references were identified by the review team as being relevant to the review question and used as a 'test library' to check whether the search strings captured the expected studies. The test library did not consist entirely of studies that would be included at every stage of the systematic review and data extraction. Instead, there were some studies which were expected to be captured in the search but did not include a suitable intervention, or were not primary literature. Whilst it was not required for the database search to capture these articles, it was a valuable test of how specific the search strings were to the subject area whilst retaining the sensitivity required to capture all available literature. The balance between specificity and sensitivity is key to achieving a comprehensive search whilst avoiding capturing too many spurious hits. The search string employed (see Additional file 1), consisting of the terms listed above, was the last of 16 iterations and was arrived at using the test library to evaluate the search results returned by Web of Knowledge.

\section{Publication databases searched}

The search included the following computerized databases:

- Web of Knowledge

- Scopus

- Agricola

- CAB Abstracts

- PubMed

- Econlit

- Directory of Open Access Journals (DOAJ)

- LILACS (Latin American \& Caribbean Health Sciences Literature - Spanish language) 
A supplemental search was undertaken using an expanded set of intervention and outcome terms to test how many extra relevant articles they might provide. The following intervention and outcome terms were entered into Web of Knowledge, and articles obtained were assessed as above.

Exposure: ecotourism, eco-tourism, eco tourism, wildlife tourism, trophy hunting, conservation corridor*, community conservanc* Outcomes: natural resource, farm, enterprise*, human AND migration, gender, discriminat*

\section{Internet searches conducted}

Internet searches using online search engines were not performed as part of this review. It was felt that the non-transparent and transient nature of search engine functionality would result in an unacceptable lack of replicability. We therefore focussed on an extensive search of specialist sources (see next section) to identify grey literature and reduce possible publication bias.

\section{Specialist searches}

A list of thirty-three relevant stakeholder organisations was identified and their websites searched by members of the qualitative and quantitative review teams and/or contacted by email with a request for relevant literature (Table 1).

Websites were searched using a hierarchical approach, from the original search string down to individual words. Where this was not possible, the following individual terms were searched; national park*, protected area*, and reserve $^{*}$. Boolean operators and wildcards were used where possible. All returns were assessed except where searches resulted in large numbers of results (i.e. > 100) when the first 50 returns were scanned for relevance. In each case, results were assessed at title and then full text, for relevance. Full texts were visually scanned for relevant data, along with within-document searches for the following terms; park, protect, and reserve. Due to the disparate modes of operation of the website search engines, a wide range of approaches was necessary and is documented in Additional file 3 .

\section{Bibliographic searches}

Where studies identified in the search used data reported in earlier primary literature, the original reference was sought and included in the data extraction process. These references were catalogued in a separate Endnote library. Five key reviews were identified from the above searches. The references within the five relevant reviews were examined to identify pertinent articles for inclusion in the review (for details, see Additional file 4). These references were assessed at title, abstract, and full text level where not already included in the above database and website searches.

\section{Search update}

In May 2013 an update to the searches was undertaken to supplement the review with all relevant evidence published since the original search was carried out in October/ November 2011.

The original search string (October 2011) was combined with the supplemental search string (March 2012) and entered into the database Web of Knowledge. Details of this search string and the number of hits returned can be found in Additional file 1.

\section{Screening}

\section{Inclusion criteria}

The following inclusion criteria were applied to those articles captured by the search.

Populations Human populations/communities currently or previously living in or near terrestrial PAs. In order to avoid subjectivity in deciding what is 'local', studies were included if the subjects were any type of community within the same country as the PA(s) that formed the focus of the paper.

Exposure Establishment/implementation, presence or change in status of terrestrial PAs areas with IUCN classifications I-VI as defined in the World Database of Protected Areas. Specifically, studies were included only where the collection of data on the impacts of PAs was undertaken during or after 1992. This included changes in status of PAs. The impacts of individual PAs were treated independently where possible but in some cases the studies looked at multiple PAs in such a way as to prevent the separation of impact. Projects that were established within or surrounding PAs as a result of the existence of that PA were also included.

Types of study Studies were considered for inclusion in this review if they satisfied one of the following criteria:

a) Evaluate the impact of PAs on human well-being (outcome evaluations which use the following study designs with appropriate comparators (i.e. a comparable state with which the intervention or exposure can be compared): Randomised Controlled Trials (RCTs) / Controlled Trials (CTs), control-intervention site comparisons, interrupted time series, Before-After/Control-Intervention (BACI) designs);

b) Reported economic valuation of welfare changes in monetary terms: based on stated or revealed preferences or production function approaches, in 
Table 1 Relevant stakeholder organisations and their websites searched for relevant literature and contacted with a request for information

\begin{tabular}{|c|c|c|}
\hline Organisation & Web Site & Email \\
\hline $\begin{array}{l}\text { Asociación Campesina e Indígena de Agroforestería } \\
\text { Comunitaria Centroamericana }\end{array}$ & http://www.acicafoc.org/ & Yes \\
\hline Conservation International & http://www.conservation.org & Yes \\
\hline Cultural Survival & www.culturalsurvival.org/ & Yes \\
\hline Department for International Development & http://www.dfid.gov.uk/ & Yes \\
\hline Development Experience Clearinghouse (DEC) & http://dec.usaid.gov/index.cfm & Yes \\
\hline EcoTerra & www.ecoterra.net/ & Yes \\
\hline Eldis (Livelihoods Connect) & http://www.livelihoods.org & Yes \\
\hline Environment Knowledge Hub & http://ekh.unep.org/ & No \\
\hline European Tropical Forest Research Network & http://www.etfrn.org/etfrn/index.html & Yes \\
\hline First Peoples Worldwide & www.firstpeoplesworldwide.org/ & Yes \\
\hline Food and Agriculture Organisation & http://fao.org & Yes \\
\hline Forest Peoples Programme & http://www.forestpeoples.org/ & Yes \\
\hline GEF Evaluation Office & http://www.thegef.org/gef/gef_Documents_Publications & Yes \\
\hline GEF Small Grants Programme & http://sgp.undp.org/ & No \\
\hline Indigenous Knowledge & http://indigenousknowledge.org/ & Yes \\
\hline International Fund for Agricultural Development & http://www.ifad.org/ & Yes \\
\hline International Institute for Environmental Development & http://www.iied.org & Yes \\
\hline International Union for Conservation of Nature & http://www.iucn.org & Yes \\
\hline Nature Valuation and Financing Network & http://www.naturevaluation.org & Yes \\
\hline Overseas Development Institute & http://www.odi.org.uk/ & Yes \\
\hline Pacific Forestry Centre & http://www.pfc.cfs.nrcan.gc.ca/ & Yes \\
\hline Poverty and Conservation & http://povertyandconservation.info & Yes \\
\hline Poverty-Well-being Platform & www.poverty-well-being.net & Yes \\
\hline RECOFTC - The Center for People and Forests & http://www.recoftc.org/site/ & Yes \\
\hline Rights and Resources Initiative & http://www.rightsandresources.org/ & Yes \\
\hline Survival International & www.survivalinternational.org/ & Yes \\
\hline Tropenbos International & http://www.tropenbos.org/ & Yes \\
\hline United Nations Development Programme & http://www.undp.org/ & Yes \\
\hline United Nations Environment Programme & http://www.unep.org/ & Yes \\
\hline United States Agency International Development & http://www.usaid.gov/ & Yes \\
\hline Waldbau-Institut, University of Freiburg & http://www.waldbau.uni-freiburg.de/ & Yes \\
\hline World Bank & $\begin{array}{l}\text { http://web.worldbank.org } \\
\text { (http://documents.worldbank.org/curated/en/home) }\end{array}$ & Yes \\
\hline World Conservation Monitoring Centre & http://www.unep-wcmc.org/ & Yes \\
\hline
\end{tabular}

which the comparator may be modelled and/or implicit in survey responses (hypothetical);

c) Sought to identify PA factors that influence human well-being or

d) Sought evidence to support explanations or meaning of impact from people's views about PAs and human well-being, and observations or related documentary analysis

We included studies that obtained data through direct measurement, self-reported measures by respondents, and other data sources that were not accessible, but the methods for which were adequately reported (such as national census data).

\section{Additional inclusion criteria for quantitative evidence}

Comparators Comparators were classified as temporal, spatial or modelled/hypothetical. Temporal comparators included time series, before and after, change over time (i.e. single time point), and reported/perceived changes. Spatial comparators included dichotomous (i.e. inside/outside and near/far) and continuous (i.e. linear distance) variables. BACI comparators included both spatial and temporal comparisons. Studies may account for confounding variability 
between comparator and exposure populations by using matching techniques, testing for differences in confounding variables, or including these variables in statistical models.

Whilst economic valuation studies may lack beforeafter or matched site comparators, economic valuations are inherently relative to a counter-factual (which may be modelled or subjective). Not all of these inherent comparators are suitable for this review, however. Many studies purport to value a PA. However, on closer inspection, they are often valuing the benefits of the ecosystem-protected area complex as a single entity, and may present no information with which to estimate the value of the ecosystem in the absence of the PA as an appropriate comparator. These studies were rejected on the grounds that they lack an appropriate comparator (the comparator is usually the complete and sudden non-existence of the ecosystem, which is deemed inappropriate for the purposes of this review). Our minimum inclusion criteria are that the study makes some attempt to model what would happen to the ecosystem in the absence of the PA, for example, estimating rates of degradation before the PA was established and extrapolating these into the future to calculate that portion of the total value of the site which is attributable to the PA. Other economic valuation studies may value opportunity costs of the PA, and in these instances the instantaneous loss of access to resources following establishment of the PA constitutes an appropriate comparator.

Outcomes Specific human-well-being indicators linked to those broad questions set out in the Background section (e.g. Livelihood strategies, social capital, empowerment, human rights, access to ecosystem services and natural resources). Examples are measures of income, education, health and other mainstream socio-economic indicators; World Bank and United Nations Development Programme (UNDP) human development index measures and measures that consider health, longevity, education, gender equity, food security, livelihood diversity, subjective/reported measures of well-being, resilience, measures of social capital, and indicators of human rights.

\section{Additional exclusion criteria for qualitative evidence}

Studies of people's views were excluded if they: focused solely on the development or validation of a measurement tool without also presenting views separately from the validation of the tool, or reported trials or other outcome evaluations, unless it was clear from the abstract that they collected data about views as part of a process evaluation.

\section{Preliminary screening process}

Articles captured by the searches were stored in an Endnote library. Replicates were removed and titles examined for relevance to the inclusion criteria. Potentially relevant titles were then separately screened as to whether they contained evidence of people's views and/or quantitative evidence of impact as described in the following sections.

\section{Screening articles for qualitative evidence}

Following preliminary screening by title (3.3.2) articles were screened for evidence of people's views of PAs by abstract and then full text. Any articles missing an abstract were accepted for full text screening. The inclusion and exclusion criteria were re-applied to the full texts $(70.3 \%$ were obtainable) and those that did not meet inclusion criteria were excluded. A coding tool was developed in order to ascertain what relevant information was held within articles (see Additional file 5). 'EPPI-Reviewer' (Thomas et al. 2010) software was used for screening using a single web location to house the documents and monitor progress of the review. Rejected articles and their corresponding reasons are listed in Additional file 6.

The systematic search and screen identified many studies relevant to the review question. The studies identified covered a broad range of geographical areas, types of PAs, and sampled a diverse population group using a variety of qualitative research methods. The characterisation of studies by methodological and contextual features provided the starting point for deciding which studies to include in the qualitative synthesis (see section Studies included in the qualitative synthesis for further details).

Descriptive information about included studies was collected and presented as a 'systematic map' of research into explanations and meaning of impact to people living in or near PAs. The 'map' provided a basis for informed discussion and decision making between both review teams about the focus of the qualitative synthesis which provides a detailed investigation of a more focused subset of this wider literature. As the synthesis was focused and narrowed down, a second set of inclusion criteria was developed and applied to the studies initially identified.

Understanding perceptions of the impact of protected areas requires appropriate research methods and full reporting of context. Further, understanding the meaning of protected areas for well-being requires qualitative data collection methods that allow people to express their views freely rather than merely responding to predetermined categories, and where the analysis provides 'thick' or 'rich' findings. 'Thick' findings have been defined as a rich, detailed description of specifics (as opposed to summary, standardization, generalization, of variables); "it captures the sense of what occurred and the drama of events, thereby permitting multiple interpretations" [14]. We therefore selected for in-depth review those studies using qualitative research methods which elicited the views of people living in or near protected areas where:

- The IUCN category, and the date this was assigned, was known 
- The methods and date of data collection were clearly reported

- The analysis produced 'thick' or 'rich' findings

- Findings were linked to specific people (e.g. distinguishing ethnicities, employments or locations)

\section{Screening articles for quantitative evidence}

Following preliminary screening by title (3.3.2) articles were screened for quantitative evidence of impact by abstract and then full text. Any articles missing an abstract were accepted for full text screening. Where information in titles or abstracts was insufficient, articles were accepted for screening at full text.

A kappa test for consistency of decision regarding inclusion/exclusion at abstract level returned a moderate level of agreement $(n=100$, kappa $=0.442,95 \%$ C.I. 0.270 to 0.614) between two independent reviewers (SED and ASP). Twenty-eight studies were treated differently by the reviewers and these were re-read and the reasons for inclusion or exclusion by each reviewer discussed. These discussions were used to come to consensus on the interpretation of the titles and abstracts and informed the subsequent inclusion process.

Full text screening resulted in the rejection of articles that were not pertinent to the review in hand. Reasons for exclusion were recorded (Additional file 6). Relevant reviews were identified for use in the bibliographic searching phase described in Section 3.2.

We attempted to obtain all articles deemed relevant during abstract-level screening, for assessment at full text. Those articles that we failed to obtain within the limitations of time and resources of the review are listed in Additional file 2.

\section{Qualitative synthesis of explanations and meaning of impact}

There are three distinctive approaches to synthesising findings from research: aggregating the findings of very similar studies where the key concepts are clearly defined in advance; configuring the findings of dissimilar studies by investigating the implications of the differences in their methods, context and findings in order to define key concepts and develop theoretical understanding; and a combination of the two which can be applied where studies have important differences, but nevertheless share some important key concepts of well-being [15].

We chose this third approach because some (but not all) key concepts were decided and defined in advance: PAs with their clearly defined categories; populations and outcomes of interest. Despite these concepts being identified by the funders, a synthesis method was required that also allows new concepts to emerge from the data in order for the impact on human well-being to be understood from the perspective of people living in or near PAs. Framework synthesis does this because dimensions of a framework for structuring the synthesis can be identified by policy interests, research interests and concepts emerging from the data [16]. Framework synthesis also takes into account differences in context such as the different countries hosting PAs, the different categories of PA and the different times and stages in their history that each area was studied. Based on framework analysis of primary research data [17], an initial conceptual framework, either built from assumptions held by stakeholders or borrowed from related bodies of knowledge, evolves during the synthesis as the reviewers become more familiar with the literature being reviewed [16]. The five neat stages of framework analysis described for primary research [18] are in practice a more iterative process for making sense of a pile of studies [19]. The process starts by delving into the abstracts then full texts to see the key issues and recurrent themes, some of which emerge from the data while others are purposely sought in response to the review question or prior knowledge. Once most of the key themes are identified, each study is coded with themes and the literature is sub-divided into sections for in-depth analysis. The conceptual framework is refined in light of these themes, and coding continues iteratively but systematically as the framework becomes increasingly coherent and simultaneously accommodating of the available data. Subsections of the framework shape data tables under key themes in order to develop concise summaries. Conclusions are drawn from the themes and associations between them.

\section{Data extraction strategy}

Included studies were inspected in detail for the focus of their findings. The coding framework described above (Additional file 5) was applied to each study. This framework covered: health (mental health, physical health, safety and wildlife conflict); socio-economic position (livelihood strategies, social capital, human rights, empowerment, environmental capital, governance); inequalities; and context. Study text was extracted in the form of: participant data (e.g. direct quotes); authors' descriptions of findings; and authors' conclusions, implementations and recommendations. The coding framework was tested by two or more reviewers independently coding successive studies, comparing and discussing how they applied the codes, and refining the framework and definitions of codes until a shared understanding was achieved across the team.

\section{Synthesis}

Synthesis began by developing the initial key concepts into a coherent framework that could accommodate the main approaches to establishing and maintaining PAs. Figure 1 illustrates two contrasting approaches to terrestrial PAs. The first is governance models that are imposed 


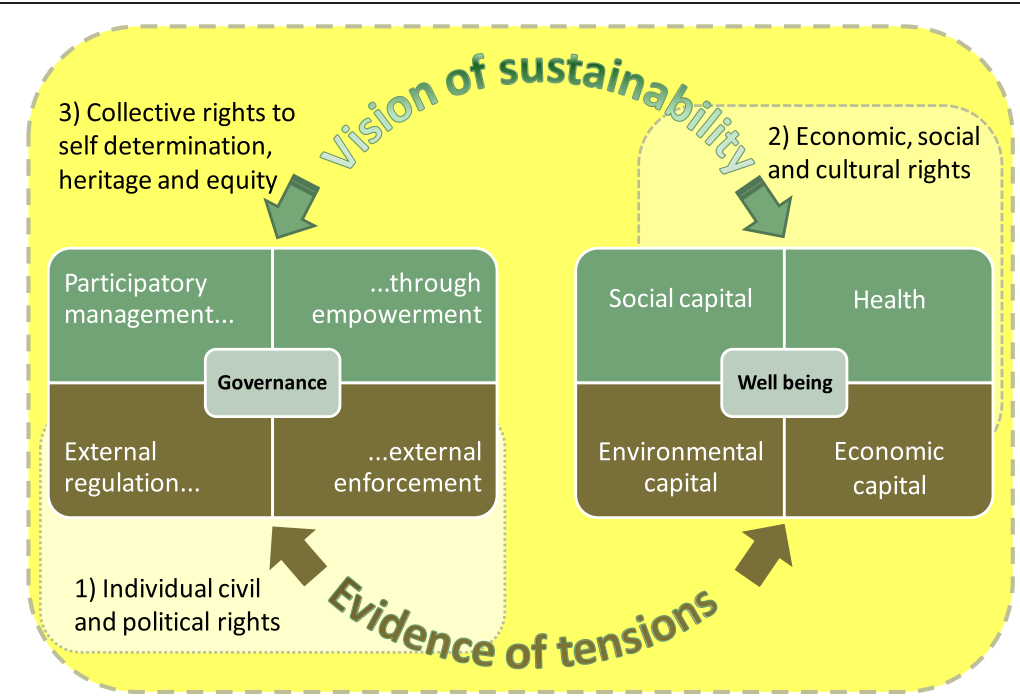

Figure 1 Conceptual framework for structuring the qualitative synthesis.

and enforced by external authorities and the second is participatory approaches such as community-based natural resource management. The latter has been developed in response to tensions raised by restrictions and resettlement having a deleterious impact on economic and environmental capital (agriculture, logging, tourism) and social capital (family and community relationships). In contrast, participatory approaches, within a regulatory framework, seek a vision of sustainability through building on social capital and good health. That vision of sustainability may be on a small scale, such as with IUCN category VI PAs, where the aim is sustainability within the boundaries. Alternatively it may be on a wider scale, across IUCN categories I or II and the wider area. These models are set against a backdrop of an evolving consensus about human rights that began with protecting individual civil and political rights, followed by the support for economic, social and cultural rights and then setting these rights within a broader framework to harness the combined efforts of individuals, states and other bodies to build collective rights to self determination, heritage and equity [20]. This synthesis aims to assess the extent to which these models and their anticipated impacts are supported by perceptions of impact on human well-being held by people living in or near protected areas and others working alongside them.

The framework was populated with research findings by dividing the studies into three overlapping subsets whose findings clearly addressed: participation (human rights, empowerment and governance), health (physical, mental and safety) and socio-economic position (wildlife conflict, livelihood strategies, social capital and environmental capital). Each set was taken by a different member of the team to seed the synthesis with studies focussing on findings in one of these three areas. Understanding the context of each study and the interplay between different concepts required reviewers to return to the full report of each study, alongside the text extracted for each code, and distil the key study characteristics and findings. The resulting coherent text was placed appropriately within the emerging framework (Figure 1). Where studies contributed substantial findings to different elements of the framework, these findings were distributed across the framework to allow each element to draw on all relevant studies. As the framework became more coherent with growing understanding of the relationships between its different elements, the elements were reordered to present governance issues first, and then well-being issues. The findings were summarised for each element of the framework, taking into account the governance and time context of the studies. The contexts of the studies contributing to the synthesis were recorded noting: the location (name and country of the protected area); IUCN category (present category, years assigned and changed); governance (national government, local government, private or community); and timing of study (year data was collected and the time lapse since the last assignment of IUCN category). Finally, all studies were revisited for additional evidence that appeared significant in light of emerging findings.

\section{Synthesis of quantitative evidence Data extraction strategy}

A preliminary data extraction phase was carried out during full text screening, where each reported study was categorised in terms of which broad outcome types were represented in the quantitative data. These outcomes were categorised according to the coding framework 
described in (and in detail in Additional file 5). Furthermore, details about the following were ascertained; the broad study methodology (i.e. self-reported data from close-ended questioning in interviews and questionnaires, direct data collection, valuation or data taken from other sources such as national census documents and similar), and whether there was a valid comparator.

Comparator categories (i.e. no comparator, before-after, inside-outside, before-after-control-impacts, econometric) were used to group the studies for a second stage of detailed data extraction. Only those studies that possessed an appropriate comparator were taken on to this second stage of data extraction, where details of the study methodology, potential effect modifiers or reasons for heterogeneity and relevant outcome data were obtained. For all outcomes, averages (means/medians) and variability measures (standard deviation, standard error and confidence intervals) were obtained where possible, along with statistical test results and sums of counts (i.e. percentages of respondents in agreement).

\section{Study quality assessment}

Details relating to study quality were extracted for each study to allow critical appraisal of relevance (external validity) with respect to the review question and reliability (internal validity). Critical appraisal was conducted in two stages. Initially assessment was made as to whether a study possessed one or more of the following: confounding variables, within-study contradictory results, inappropriate implicit comparator, inappropriate population, inappropriate spatial comparator, inappropriate statistics, inappropriate temporal comparator, inappropriate time frame, insufficient detail in methods, data linked with marine ecosystem protection, non-standardised data (incomparable), protected area establishment too recent/not yet established, data collection prior to 1992, extremely small sample size (e.g. one study, where wood extraction was quantified in only nine households with one intervention and one control village), superseded by more recent study, unbalanced questioning/questioning bias, and unmatched methods (see Additional file 6 for further detail). Studies with one or more of these characteristics were excluded from the review.

Studies accepted following this first critical appraisal stage were then scored for reliability with respect to the question using the concept of susceptibility to bias (the extent to which a study is able to yield an unbiased estimate of effect; [21]). Susceptibility to bias in study design and reporting can affect both internal validity (study quality) and external validity (study generalisability).

Susceptibility to bias scores were assigned using a repeatable, but partially subjective, set of four criteria, each assessed on a scale of 0 to 2 , and thus ranging from a minimum possible value of 0 and a maximum of 8 (Table 2). The four criteria were as follows:

i) Clarity of methods; the extent of detail given in the study's methodology regarding questionnaire design, survey implementation, replicate number and selection, and data analysis

ii) Study design; the rigour of study design in terms of replicate number and sampling, location and choice of replicates and controls, questionnaire design, and survey implementation

iii) Appropriateness of analysis; the suitability of analytical techniques such as the implementation and choice of statistics, and comprehensiveness of analysis

iv) Implementation bias; the presence of one of the following biases in measurement and analysis; Neyman bias (the intervention in question causes systematic drop-out within the population, e.g. the most affected families move away), questioning bias (unbalanced/leading questions), potentially influential variables, recall bias (response affected by memory).

Susceptibility to bias scores were then combined with an objective weighting by basic study design in order to provide a categorical assessment (high, medium or low) of susceptibility to bias (Table 3).

\section{Potential effect modifiers and reasons for heterogeneity}

Data on potential effect modifiers that were extracted from articles, included the following variables; protected area studied, country, IUCN category, protected area size, date of establishment, and history of protection prior to formal establishment, and residence history and ethnicity of the study population. Where sufficient information about IUCN category, protected area size and year of establishment was not presented in the article itself, data were supplemented from the World Database on Protected Areas (WDPA) (http://www.protectedplanet.net/).

\section{Data synthesis and presentation}

Extracted data were presented in narrative synthesis tables that summarise the studies' aims, methodology, study population, and results (Additional file 7). No further quantitative synthesis of data on outcomes was possible with the available data.

\section{Results}

\section{Review descriptive statistics}

The main literature search was conducted between 11 October and 14 November 2011 with an update carried out in May 2013. 
Table 2 Scoring criteria for subjective assessment of susceptibility to bias

\begin{tabular}{|c|c|c|c|}
\hline Assessment criteria & 0 & 1 & 2 \\
\hline Clarity of methods & $\begin{array}{l}\text { All sample sizes provided, selection } \\
\text { method described, questionnaire } \\
\text { design fully disclosed }\end{array}$ & $\begin{array}{l}\text { Some information regarding sample } \\
\text { size, selection method, or } \\
\text { questionnaire design provided }\end{array}$ & $\begin{array}{l}\text { Sample size not given in full, sample selection } \\
\text { not stated, questionnaire design not discussed }\end{array}$ \\
\hline Experimental design & $\begin{array}{l}\text { Good sample size, appropriate sampling } \\
\text { regime, control and intervention well } \\
\text { matched, survey appropriately } \\
\text { implemented }\end{array}$ & $\begin{array}{l}\text { Low effective sample size, poorly } \\
\text { randomised design, control and } \\
\text { intervention choice not ideal }\end{array}$ & $\begin{array}{l}\text { Very small sample size, pseudoreplication, } \\
\text { non-random sample selection, control and } \\
\text { intervention poorly matched/low } \\
\text { consistency in sampling over time }\end{array}$ \\
\hline $\begin{array}{l}\text { Appropriateness of } \\
\text { analysis }\end{array}$ & $\begin{array}{l}\text { Confounding variables accounted for, } \\
\text { appropriate metrics reported }\end{array}$ & $\begin{array}{l}\text { Confounders only partly } \\
\text { accounted for/only some low } \\
\text { risk confounders ignored }\end{array}$ & $\begin{array}{l}\text { Significant confounding variables unaccounted } \\
\text { for, inappropriate metrics reported, incorrect } \\
\text { statistical analysis }\end{array}$ \\
\hline Implementation bias & No identifiable bias reported/evident & $\begin{array}{l}\text { Low level bias present but ignored/ } \\
\text { strong bias accounted for }\end{array}$ & Strong bias present and unaccounted for \\
\hline
\end{tabular}

The main search returned 13,248 articles (following removal of duplicate and marine-oriented articles) and title-level screening left a total of 1,710 articles. The search update returned 3,122 articles (following removal of duplicates) and title-level screening left a total of 204 additional articles. From this point forwards, screening at abstract was independent for the two review sections.

\section{Qualitative evidence}

Abstract screening for the qualitative review resulted in the final inclusion of 30 studies as illustrated in Figure 2. Four additional studies were included in an identical process following the search update in May 2013.

\section{Quantitative evidence}

Abstract screening for the quantitative review resulted in the final inclusion of 79 studies as illustrated in Figure 3.

In addition to the above searches 2,345 articles were identified by the supplemental search conducted on 23 March 2012: 535 remained following title-level screening, and 171 following abstract-level screening. Twelve studies from this supplemental search were included following critical appraisal.

Relevant organisation website searches conducted between 6 and 29 March 2012 yielded 94 relevant articles for the quantitative review. In addition to these searches, 50 relevant articles were identified through bibliographic checks and secondary sources, yielding a total of 1,164 potentially relevant articles. Of these articles, $76 \%$ were retrievable for full text assessment, whilst 275 were

Table 3 Categorization of susceptibility to bias

\begin{tabular}{llll}
\hline & \multicolumn{3}{c}{ Susceptibility to bias score } \\
\cline { 2 - 4 } Comparator & $\mathbf{0 - 2}$ & $\mathbf{3 - 5}$ & $\mathbf{6 - 8}$ \\
\hline RCT $^{*}$ (randomised control trials) & Low & Low & Medium \\
\hline BACl (before-after-control-intervention) & Low & Medium & High \\
\hline $\begin{array}{l}\text { Control-intervention/Before-after/ } \\
\text { Econometric }\end{array}$ & Medium & High & High \\
\hline
\end{tabular}

*There were no RCT studies and this line is only provided for a theoretical perspective unobtainable within the timeline and resource limitations of the review. These numbers are represented visually in Figure 3. One hundred and fifty-seven of the articles identified in the May 2013 search update were obtainable, although five of these were in Chinese and could not be assessed (see Additional file 2).

Following full text assessment 177 articles from the original search, 16 from the supplemental search and 17 from the update met the inclusion criteria and were subsequently critically appraised. Following critical appraisal, articles/studies were rejected at full text for a wide range of reasons (see section Study quality assessment). A list of these articles and associated reasons is provided in Additional file 6 . A final set of 70 articles was included, reporting on 79 studies and these are listed in Additional file 7.

\section{Comparison of included studies from the two review processes}

The following statistics describe the 30 studies on qualitative evidence of people's views and 79 on quantitative evidence of impact included in the synthesis.

Study location Figure 4 displays the countries from which data were collected for the included studies from the two processes. Frequently studied countries include Cameroon, China, India, and Uganda for the qualitative synthesis and India, Nepal, South Africa and Uganda for the 'quantitative impacts' review.

Category of PA studied The IUCN categories of the PAs (see below) examined in the included studies are shown in Figure 5. PAs predominantly fell under category II in both reviews, with 17 percent either unreported by WDPA or not present in the database for the 'quantitative impacts' review (NB this group includes only 'not applicable' for the qualitative synthesis, since studies were only included in this review if the protected area IUCN category was stated). A post-hoc decision to exclude category III PAs was made as they are small in number and somewhat atypical of the set of categories 


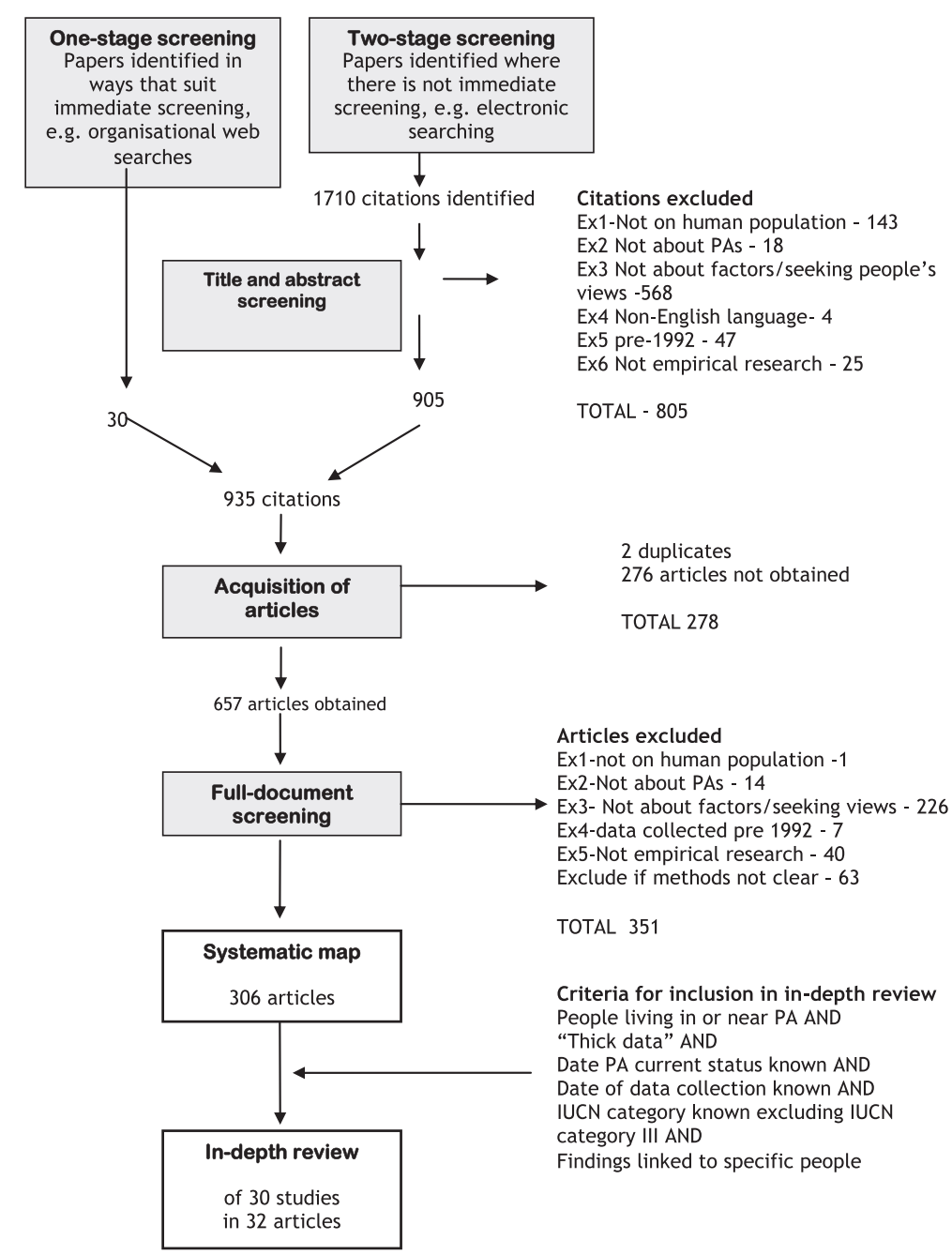

Figure 2 Number of articles and studies at progressive stages of inclusion for qualitative synthesis.

(specifically protecting natural monuments). Similar patterns were observed in both review processes Figure 6 displays PA establishment year, showing a peak in new PAs centered around the 1980s.

IUCN Protected Area Categories are described as follows:

Ia Strict Nature Reserve - protected from all but light human use in order to preserve all geological and geomorphological features of the region and their biodiversity, which is often dense and restricted to exclusively to scientific monitoring, study or education. Occasionally Strict Nature Reserves are of spiritual significance to surrounding communities in which case the people are generally allowed to continue the practice of their faith and may be directly involved in the area's conservation and management objectives, though perpetual human intervention would more suitably be allocated to categories IV or V.
Ib Wilderness Area - generally larger than Strict Nature Reserves, the main objectives of these areas is to provide an environment in which biodiversity and ecosystem processes (including evolution) are allowed to flourish or experience restoration if previously disturbed by human activity. Human use is limited, often allowing only those who are willing to travel of their own accord rather than via established touristic activities. Wilderness areas can be classified as such only if they are devoid of modern infrastructure, although they allow human activity to the level of sustaining indigenous groups living wilderness-based lifestyles.

II National Park - provide protection for functioning ecosystems (similar to the objectives of Wilderness Areas), but tend to be more lenient with human visitation and the supporting infrastructure. National Parks are managed in a way that may contribute to local economies through promoting educational and 


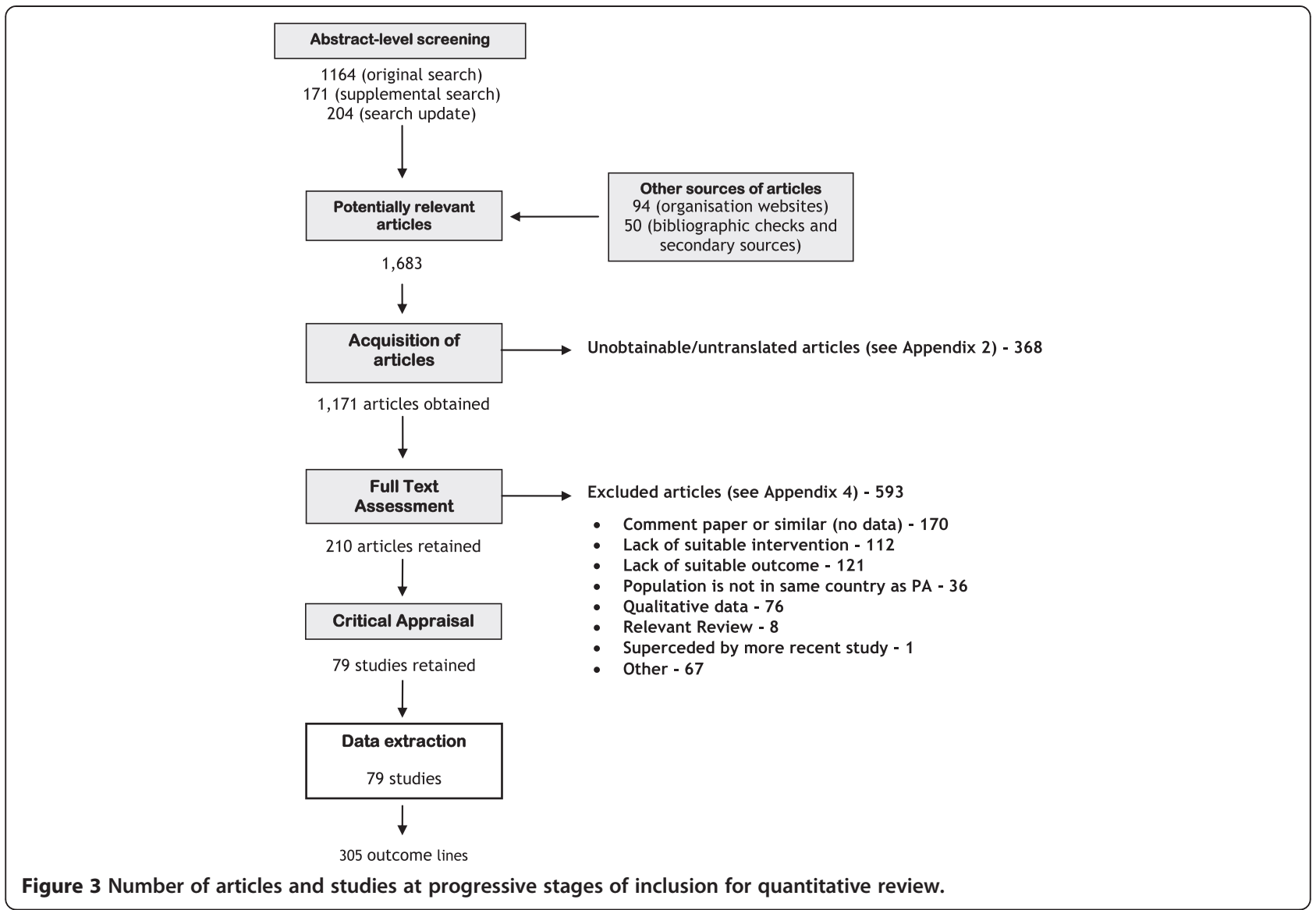

recreational tourism on a scale that will not reduce the effectiveness of conservation efforts. The surrounding areas of a National Park may be for consumptive or non-consumptive use, but should nevertheless act as a barrier for the defence of the protected area's native species and communities to enable them to remain sustainable in the long term.

III Natural Monument or Feature - comparatively smaller areas, specifically allocated to protect a natural monument and its surrounding habitats. Natural Monuments or Features can be natural in the wholest sense, or include elements that have been influenced or introduced by humans. The latter should hold biodiversity associations or could otherwise be classified as a historical or spiritual site, though this distinction can be quite difficult to ascertain. As such, the classification then falls into two subcategories, those in which the biodiversity in uniquely related to the conditions of the natural feature, and those in which the current levels of biodiversity are dependent on the presence of the sacred sites that have created an essentially modified ecosystem.

IV Habitat/Species Management Area - focus on more specific areas of conservation in correlation to an identifiable species or habitat that requires continuous protection. These protected areas will be sufficiently controlled to ensure the maintenance, conservation and restoration of particular species and habitats - possibly through traditional means and public education of such areas is widely encouraged as part of the management objectives. Habitat or Species Management Areas may exist as a fraction of a wider ecosystem or protected area and may require varying levels of active intervention including - but not limited to - the prevention of poaching, creation of artificial habitats, halting natural succession and supplementary feeding practices.

VI Protected Landscape/Seascape - cover entire bodies of land or ocean which engage a range of forprofit activities within the management plan. The main objective is to safeguard regions that have built up a 'distinct character' in regards to their ecological, biological, cultural or scenic value. Protected Landscapes and Seascapes allow a higher level of sustainable interaction with -surrounding communities (such as traditional agricultural and forestry systems) and should represent an integral balance between people and nature. 


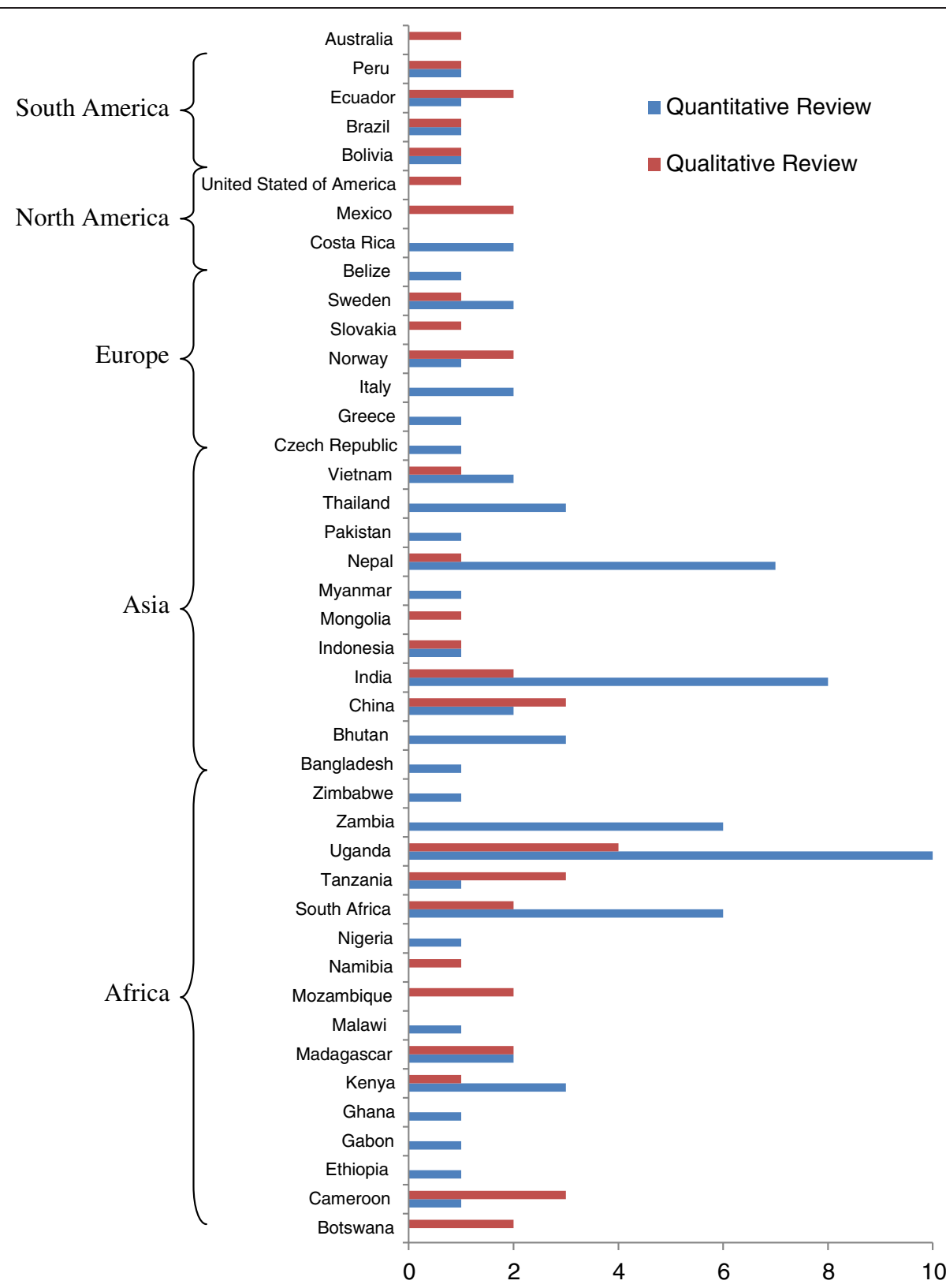

Figure 4 Frequency of countries, arranged by continent, hosting the protected areas within included studies for the two reviews.

VI Protected area with sustainable use of natural resources - based on a mutually beneficial relationship between nature conservation and the sustainable management of natural resources in correspondence the livelihoods of surrounding communities. A wide range of socio-economic factors are taken into consideration in creating local, regional and national approaches to the use of natural resources. Though human involvement is a large factor in the management of these protected areas, developments are not intended to allow for widescale industrial production. Governance has to be developed to adapt to the diverse and possibly growing range of interests that arise from the production of sustainable natural resources. (Text adapted from United Nations Environment Programme (UNEP) World Conservation Monitoring Centre (WCMC) website (http://www. unep-wcmc.org/iucn-protected-area-managementcategories_591.html; accessed 03/10/2012)

Study timescale Figure 7 displays the survey years for the included studies for both reviews. A significant number of studies failed to report the survey year (applicable 


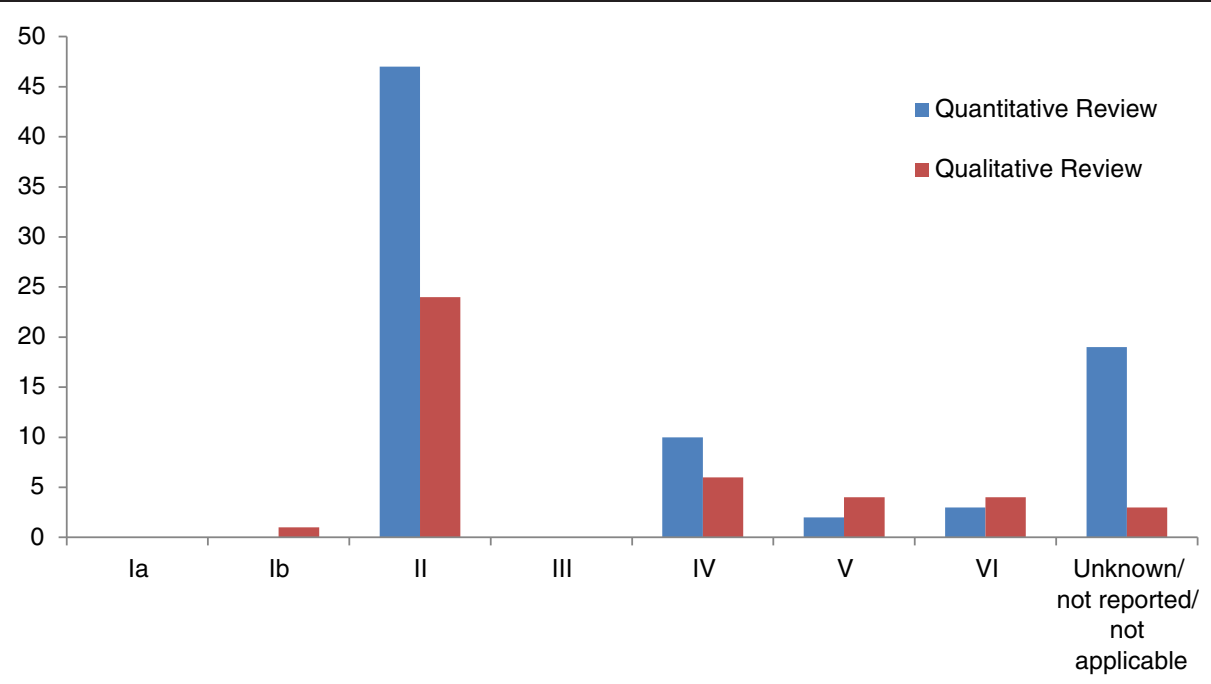

Figure 5 Frequency of all named PAs by IUCN category within included studies.

only to the 'quantitative impacts' review, since this was an exclusion criterion for the qualitative synthesis). The reduction in the number of surveys from 2005-2006 for both reviews likely relates to a publishing delay.

\section{Further descriptive statistics for the review of quantitative evidence of impacts}

Study comparator Figure 8 displays the range and frequency of comparators used in included studies. Spatial comparators (site comparators and linear distance) were common, along with reported change over time. 'Before and after, control intervention' (BACI) studies were very rare, with only one instance of a full BACI study.
Measured outcome Within the five key themes described in Section 1 (Livelihood Strategies, Human Rights, Social Capital, Empowerment, and Access to Ecosystem Goods and Services), 24 individual outcomes were identified in an iterative process during screening. The reported outcomes were separated in order to facilitate the description and analysis of similar, comparable data. The frequency of these broad outcome types is shown in Figure 9. Attitude, income, and wildlife conflict outcomes were most common.

Exclusion of studies following critical appraisal resulted in several outcomes identified during full title assessment being unrepresented by data namely, 'empowerment' and 'religion and spirituality'. Other outcomes are represented in

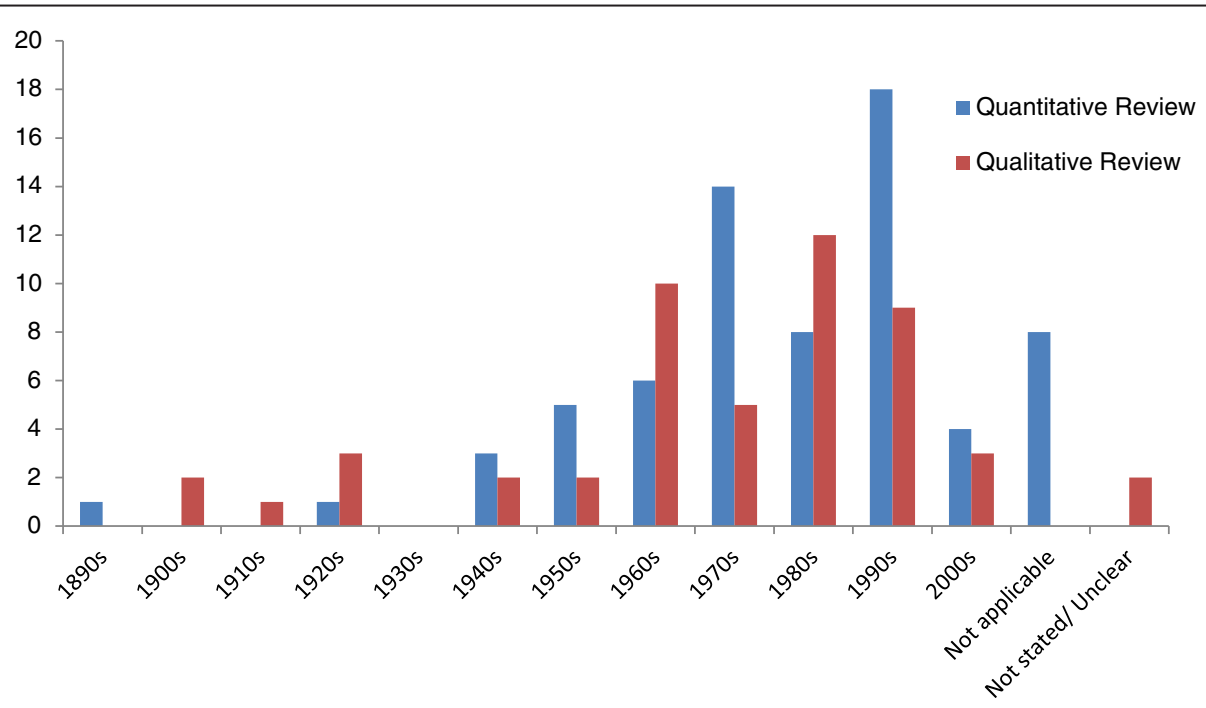

Figure 6 Frequency of year of establishment of PAs within included studies. 


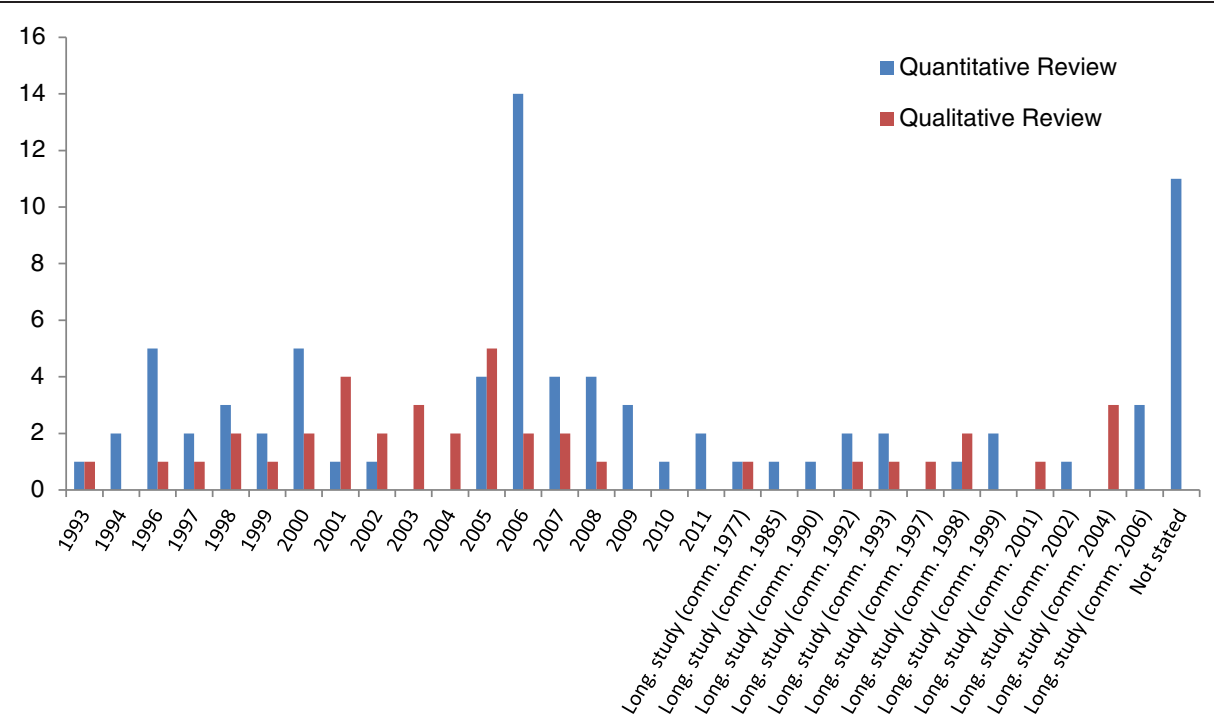

Figure 7 Frequency of year of PA survey for included studies. Surveys commence on stated year and are classified as 'longitudinal study' if survey spans more than two years.

two studies or fewer; 'gender equity', 'interactions with PA authorities', 'livelihood diversity', 'medicinal plants/animals', 'protect for future', and 'resettlement and displacement'.

Methodology of data collection Figure 10 displays the frequency of different data sources in included studies, showing that self reported measures formed the majority of data reported (63 of the 79 included studies).
Qualitative synthesis of explanations and meaning of impact Studies included in the qualitative synthesis

The criteria for selecting studies to include in the in-depth qualitative synthesis is provided in section Screening articles for qualitative evidence. Although not statistically representative of the 306 studies of people's views identified (characterised in the map see Additional file 8), studies with 'thick' data were chosen for their ability to provide explanations and meaning of impact for people living in or

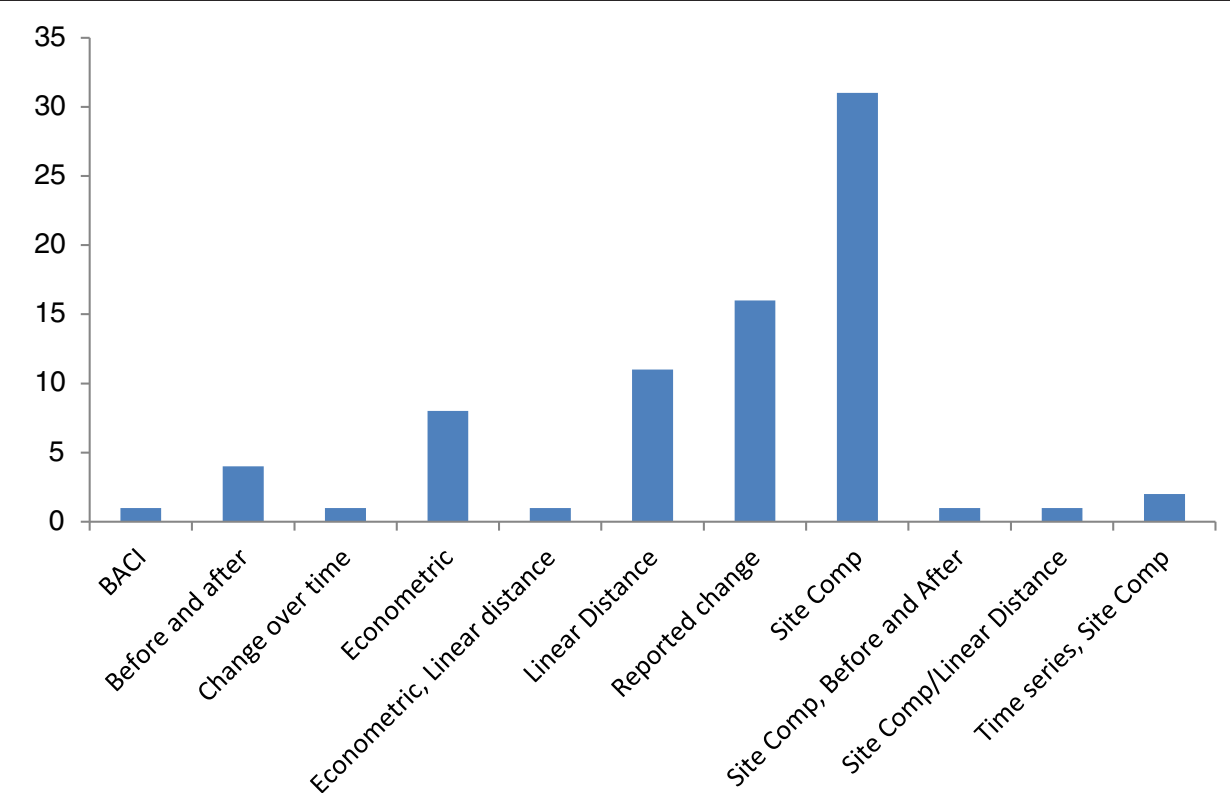

Figure 8 Frequency of comparators used in included studies. 


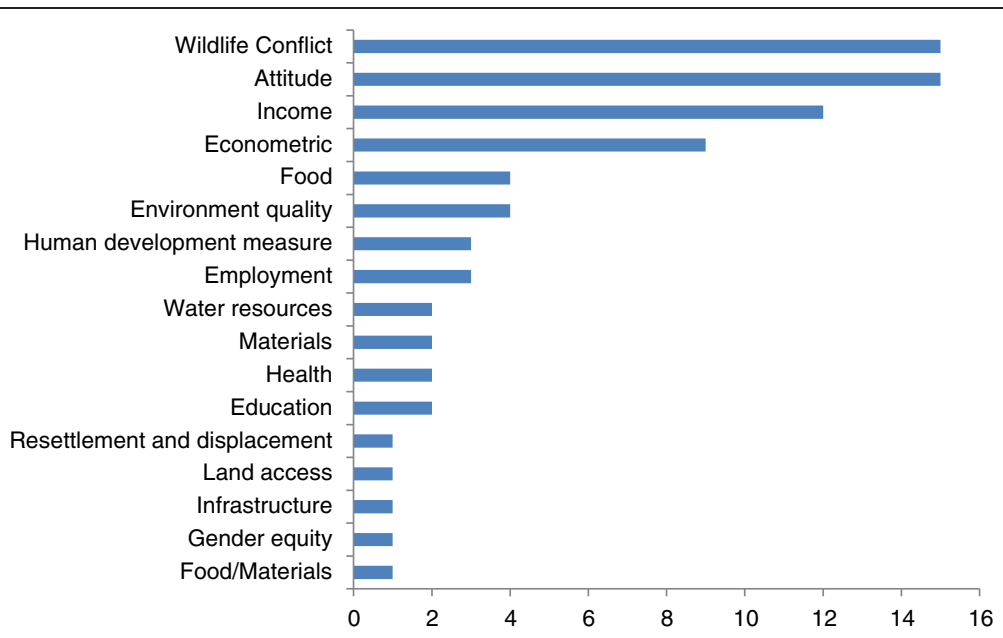

Figure 9 Frequency of broad outcome types in included studies.

near the PA. They also spanned the range of the broader literature, in terms of countries frequently studied, as described in the map. However, poor reporting makes it difficult to conduct further comparisons between the studies reviewed in-depth with the wider map in terms of IUCN categories and key dates.

The vast majority of included studies were by academic authors, with a small minority from NGOs, commercial organisations and protected area authorities. These different perspectives are likely to shape the focus of studies and, consequently, their findings.

Residents' views were most often sought for IUCN category II, where there are strict restrictions on settlements, but rarely for IUCN category VI, where the aim is to balance the aims of conservation and the needs of the local population. Leaders' views were most often sought about IUCN category I protected areas, which does not allow human settlements. There was also a noticeable absence of studies addressing the views of protected area authorities or employees in category IV (similar to the map).

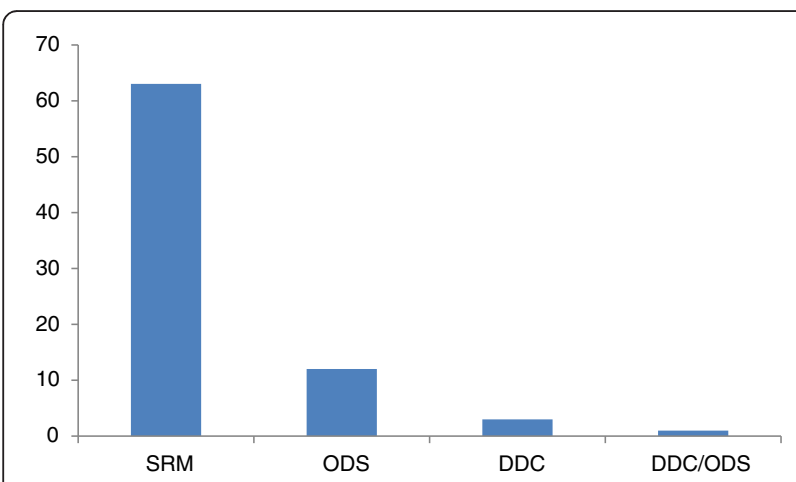

Figure 10 Frequency of data sources in included studies. DDC, direct data collection by study authors; ODS, other data sources; SRM, self-reported measure via survey responses.
The other stakeholders not well represented in the studies presenting 'thick' findings are visitors to protected areas. Studies seeking visitors' views often did so using highly structured questionnaires that provided little understanding of what protected areas mean to them. The one study of visitors included in the synthesis was set in a category IV protected area, so was not typical of most visitor surveys which, in the map, were most often used in category II.

This literature is thus a likely source for understanding people's views, and setting those views in context, across the IUCN categories, although less so for category IV.

\section{Summary findings of qualitative synthesis of explanations and meaning of impact}

This section presents a summary of a full synthesis of findings from studies that provide explanations or explore the meaning of the impact of protected areas on people living in or near PAs. The full synthesis can be found in Additional file 9. It also draws on the perspectives of other stakeholders relevant to those experiences and relevant policy and historical documents. The synthesis is presented within a conceptual framework that was informed by conservation policies and refined by the emerging research literature (Figure 1).

The synthesis process identified the implementation issues and characteristics of well-being that were associated by research participants with either (a) tensions arising from governance models imposed and enforced by external authorities or (b) a vision of sustainability sought through participatory management and empowerment commonly known as community-based natural resource management.

The latter approach has been developed in response to tensions raised by restrictions and resettlement having a deleterious impact on economic and environmental 
(natural) capital and social capital. Participatory approaches, within a regulatory framework, seek a vision of sustainability through building on social capital and good health. That vision of sustainability may be on a small scale, such as with IUCN category VI PAs, where the aim is sustainability within the boundaries. Alternatively it may be on a wider scale, across IUCN categories I or II and the wider area. These models are set against a backdrop of an evolving consensus about human rights that began with protecting individual civil and political rights, followed by the support for economic, social and cultural rights and then setting these rights within a broader framework to harness the combined efforts of individuals, states and other bodies to build collective rights to self determination, heritage and equity [20]. This synthesis aims to assess the extent to which these models and their anticipated impacts are supported by the perceptions of people living in or near protected areas and others working alongside them.

The characteristics of the included studies are summarised in Table 4, and their findings are subsequently synthesised in sections that match the conceptual framework (see Figure 11) to present a coherent narrative which explores key themes within governance (source of authority and nature of implementation) and then considers the impact on health and well-being in terms of environmental (natural), economic and social capital. There is necessarily some overlap between the governance issues and the impact on health and well-being. The key messages for both are presented in boxes to help readers navigate the breadth and depth of the available evidence. The contextual details of each study described in terms of IUCN Category, the date the PA was established, date of current PA status being assigned, time between current status being assigned and data collection are reported in Additional file 9. As the complexity of the links between governance and well-being precludes simple distinctions between PA approaches and their impacts, we focus first on governance and then on well-being, acknowledging that there is necessarily some overlap. The key messages for both are presented in boxes to help readers navigate the breadth and depth of the available evidence.

\section{Governance}

Matching study findings to the conceptual framework facilitated their comparison and interpretation to reveal problems and potential solutions during implementation and instances of both benefit and harm.

- Portraying longstanding residents as an environmental risk to pristine protected areas is controversial

- PA regulations can flounder as a result of inaccurate maps and poorly drafted legislation, and further confusion arises from discrepancies between state rules and local institutions and typically poor communication

- Memories of forced or induced migrations negatively influence subsequent community responses to authorities

- Inadequate or non-existent compensation is a widely held concern

- Externally imposed regulations were incompatible with traditional regulations and did not take into account cultural and social diversity; respect for the rules was greater where they were locally adapted and allowed income-generating possibilities.

- A major challenge is distinguishing subsistence activities for a sustainable environment from larger scale industrial activities.

- Success came from PAs staff having: prior experience of working with locals; clear guidelines; extensive training in community development, gender issues and a variety of participatory methodologies; meeting locals informally and working with existing kinship networks.

- All studies contributed some evidence related to governance.

Implementation problems The earliest source of tension in the life course of protected areas can be controversy about environmental risks posed by local inhabitants. Studies in Australia [22], Nepal [23], Norway [24], Mexico [25], Indonesia [26] and USA [27] revealed local inhabitants appreciating areas for their aesthetic and spiritual values as well as environmental products and economic and leisure opportunities. Mexican farmers in particular value the land for its provision of food, water, wood and other products, and they have developed farming styles along a spectrum of reciprocal relationships between man and nature between wilderness and urbanisation [28]. Diaw [29] claims that a resettlement policy to establish a IUCN II category park in Cameroon in 1961 was driven by scientific myths of a pristine forest whose protection was incompatible with indigenous residents despite historical analysis showing that the current forest structure was the result of sustained use over centuries. Economic arguments favouring resettlement were flawed, with excessively strong assumptions about tourism benefits, flood control, forest use, research discoveries, soil fertility and agricultural productivity. Similarly, in Norway residents pointed to a lack of convincing scientific evidence supporting the need for certain protective measures [24]. Residents of Utah claimed the land benefitted from how they had cared for it before it was declared a protected area (Grand Staircase Escalante National Monument, IUCN II [27]). In Norway, local tourist firms were frustrated by the lack of opportunities to play a part in the protected area management (IUCN II). They claimed that local expertise, based on generations of 
Table 4 Summary table of included studies in the qualitative synthesis

\begin{tabular}{|c|c|c|c|c|c|c|c|c|}
\hline Citation & Protected area & $\begin{array}{l}\text { IUCN } \\
\text { Category }\end{array}$ & Date PA established & $\begin{array}{l}\text { Date of current } \\
\text { IUCN category }\end{array}$ & $\begin{array}{l}\text { Years since IUCN } \\
\text { category change/ } \\
\text { establishment }\end{array}$ & $\begin{array}{l}\text { Year of data } \\
\text { collection }\end{array}$ & Governance model & Country \\
\hline Allendorf et al. (2007) & Royal Bardia NP & $\|$ & 1969 & 1976 & 14 & 1990 & $\begin{array}{l}\text { Government: federal/ } \\
\text { national }\end{array}$ & Nepal \\
\hline Almudi and Berkes (2010) & Peixe Lagoon National Park & V & 1986 & $2001-2010$ & 3 & $2005 \& 2007$ & $\begin{array}{l}\text { Cooperatively managed: } \\
\text { collaboratively }\end{array}$ & Brazil \\
\hline $\begin{array}{l}\text { Bedunah and Schmidt } \\
\text { (2004) }\end{array}$ & Gobi Gurvansaikhan & $\|$ & 1993 & 1993 & $5-7$ & $1998-2000$ & Information not found & Mongolia \\
\hline Bizikova et al. (2012) & Slovensky Raj Park & $\|$ & 1998 & 1998 & 8 & 2006 & $\begin{array}{l}\text { Government: federal/ } \\
\text { national }\end{array}$ & Slovakia \\
\hline Bolaane (2004) & Moremi Game Reserve & IV & 1965 & 1965 & 32 & $1997-2001$ & Private & Botwana \\
\hline Bruyere et al. (2009) & Buffalo Springs Samburu & $\|$ & 1985 & 1985 & 20 & 2005 & Government: local & Kenya \\
\hline \multirow[t]{3}{*}{ Castillo et al. (2005) } & El Vizcaíno & IV & 1988 & 1988 & 16 & 2004-2007 & $\begin{array}{l}\text { Government: federal/ } \\
\text { national }\end{array}$ & Mexico \\
\hline & Sian Ka'an & & 2006 & 2008 & 3 & & & \\
\hline & Monarch Butterfly & & 2008 & & $<1$ & & & \\
\hline Davis (2011) & Tarangire National Park & $\|$ & 1970 & 1970 & 35 & $2005-2007$ & Government: local & Tanzania \\
\hline Diaw (2010) & Korup National Park & $\|$ & 1961 & 1985 & 18 & 2003 & $\begin{array}{l}\text { Government: federal/ } \\
\text { national }\end{array}$ & Cameroon \\
\hline \multirow[t]{3}{*}{ First Peoples (2006) } & Mgahinga National Park & $\|$ & 1930 & 1930 & Not stated & & $\begin{array}{l}\text { Government: federal/ } \\
\text { national }\end{array}$ & Uganda \\
\hline & Bwindi National Park & & 1991 & 1991 & 14 & 2005 & $\begin{array}{l}\text { Government: federal/ } \\
\text { national }\end{array}$ & Uganda \\
\hline & Awa Forest Reserve Zone & $\mathrm{Vl}$ & 1988 & 1988 & $10-20$ & $1998-2008$ & Community: indigenous & Ecuador \\
\hline Gerritsen (2002) & $\begin{array}{l}\text { Sierra De Manantlan } \\
\text { Biosphere Reserve }\end{array}$ & $\mathrm{Vl}$ & 1987 & 1987 & $6-11$ & $1993-1998$ & $\begin{array}{l}\text { Government: federal/ } \\
\text { national }\end{array}$ & Mexico \\
\hline Hartter (2009) & Kibale national park & $\|$ & 1932 & 1993 & 16 & & & Uganda \\
\hline \multirow[t]{2}{*}{ Haukeland (2011) } & Rondane National Park & $\|$ & 1962 & 1962 & 47 & 2009 & & Norway \\
\hline & Jotunheimen National Park & $\|$ & 1980 & 1980 & 18 & & Government: national & \\
\hline Herrold-Menzies (2011) & Caohai Nature Reserve & $\begin{array}{l}\text { V Protected } \\
\text { landscape/ } \\
\text { Seascape }\end{array}$ & 1985 & 1985 & $13-16$ & $\begin{array}{l}\text { 1998-1999, 2000, } \\
2000-2001\end{array}$ & $\begin{array}{l}\text { Government } \\
\text { managed: local }\end{array}$ & China \\
\hline Hoole and Berkes (2010) & Etosha National park & $\|$ & 1975 & 1975 & 31 & $2006-2007$ & Government & Namibia \\
\hline \multirow[t]{4}{*}{$\begin{array}{l}\text { Keskitalo and Lundmark } \\
\text { (2010) }\end{array}$} & Sarek National Park; & $\mathrm{lb}$ & 1909 & 1909 & 95 years & 2001 & $\begin{array}{l}\text { Government: federal/ } \\
\text { national }\end{array}$ & Sweden \\
\hline & Stora Sjöfallet & & 1909 & 1909 & 95 years & & & \\
\hline & National Park; Abisko & $\|$ & 1920 & 1920 & 83 years & & & \\
\hline & $\begin{array}{l}\text { National Park; Pieljekaise } \\
\text { National Park; Vadvetjåkka }\end{array}$ & & 1962 & 1982 & 22 years & & & \\
\hline
\end{tabular}


Table 4 Summary table of included studies in the qualitative synthesis (Continued)

\begin{tabular}{|c|c|c|c|c|c|c|c|c|}
\hline \multirow[b]{3}{*}{ Lunstrum (2008) } & \multicolumn{8}{|l|}{$\begin{array}{l}\text { National Park; Padjelanta } \\
\text { National Park }\end{array}$} \\
\hline & \multicolumn{8}{|l|}{$\begin{array}{l}\text { Haparanda-Sandskär } \\
\text { Nature Reserve }\end{array}$} \\
\hline & Limpopo National Park & $\|$ & 1979 & 1979 & 25 & $2004-2005$ & $\begin{array}{l}\text { Cooperative: transboundary/ } \\
\text { collaborative }\end{array}$ & Mozabique \\
\hline Mbaiwa (2005) & Moremi Game Reserve & IV & 1965 & 1965 & 38 & $\begin{array}{l}1998,2001 \\
2003\end{array}$ & Private: for profit & Botswana \\
\hline Mehring et al. (2011) & Lore Lindu National Park & $\|$ & 1982 & 1982 & 24 & $2006-2008$ & $\begin{array}{l}\text { Government: federal/ } \\
\text { national }\end{array}$ & Indonesia \\
\hline $\begin{array}{l}\text { Milgroom and } \\
\text { Sperienburg (2008) }\end{array}$ & Limpopo National Park & $\|$ & 2001 & 2001 & $6-7$ & $2007-2008$ & $\begin{array}{l}\text { Cooperative: transboundary/ } \\
\text { collaborative }\end{array}$ & Mozabique \\
\hline Nguiffo (2001) & Dja Wildlife Reserve & IV & 1950 & 1950 & 51 & 2001 & $\begin{array}{l}\text { Government: federal/ } \\
\text { national }\end{array}$ & Cameroon \\
\hline Ogra (2008) & Rajaji National Park & $\|$ & 1983 & 1983 & 20 & $2003-2004$ & Information not found & India \\
\hline Ormsby and Kaplan (2005) & Masoala National park & $\|$ & 1997 & 1997 & 4 & 2001 & Private/ non-profit & Madagascar \\
\hline \multirow[t]{2}{*}{$\begin{array}{l}\text { Petrzelka and Marquart- } \\
\text { Pyatt (2013) }\end{array}$} & Grand Staircase & V & 1996 & 1996 & 0 & 1996 & $\begin{array}{l}\text { Government: federal/ } \\
\text { national }\end{array}$ & USA \\
\hline & $\begin{array}{l}\text { Escalante National } \\
\text { Monument }\end{array}$ & & & & 10 & 2006 & & \\
\hline Slater (2002) & Qwaqwa National Park & IV & 1992 & 1992 & $6-7$ & $1998-1999$ & Government: local & South Africa \\
\hline Sletten et al. (2008) & Mount Elgon NP & $\|$ & 1951 & 1951 & 47 & 2002 & $\begin{array}{l}\text { Government: federal/ } \\
\text { national + collaborative }\end{array}$ & Uganda \\
\hline $\begin{array}{l}\text { Spenceley and } \\
\text { Goodwin (2005) }\end{array}$ & Kruger National Park & $\|$ & 1926 & 1930 & $74-75$ & $2000-2001$ & Information not found & South Africa \\
\hline Songorwa (1999) & Selous Game Reserve & IV & 1905 & 1905 & 91 & 1996 & Community: local & Tanzania \\
\hline Stone and Wall (2004) & $\begin{array}{l}\text { Jianfengling National Forest } \\
\text { Park and Diaoluoshan } \\
\text { National Forest Park in } \\
\text { Hainan Province, China }\end{array}$ & V & 1986 & 1986 & 16 years & 2001 & Government: local & China \\
\hline $\begin{array}{l}\text { Strickland-Munro and } \\
\text { Moore (2012) }\end{array}$ & Purnululu National Park & $\|$ & 2003 & 2003 & 5 years & 2008 & $\begin{array}{l}\text { Government: federal/ } \\
\text { national + collaborative }\end{array}$ & Australia \\
\hline Stronza and Gordillo (2008) & $\begin{array}{l}\text { Kapawi Reserve; Madidi } \\
\text { National Park; Bahuaja } \\
\text { Sonene National }\end{array}$ & $\|$ & $\begin{array}{l}\text { Not stated Kapawi Reserve, } \\
\text { Ecuador 1995: Madidi } \\
\text { National Park 2000: Bahuaja } \\
\text { Sonene National Park, Peru }\end{array}$ & $1991-2000$ & $\begin{array}{l}\text { Not able to ascertain } \\
\text { Ecuador } 5 \text { years:, Bolivia; } \\
8 \text { years:, Peru }\end{array}$ & 2003 & $\begin{array}{l}\text { Cooperatively managed } \\
\text { Collaborative management } \\
\text { (various forms of } \\
\text { pluralist influence) }\end{array}$ & $\begin{array}{l}\text { Bolivia } \\
\text { Ecuador Peru }\end{array}$ \\
\hline Torri (2011) & Sariska Tiger Reserve & IV & 1955 & 1955 & 46 & $\begin{array}{l}2001 \text { (pilot) } \\
2007\end{array}$ & $\begin{array}{l}\text { Government: federal/ } \\
\text { national }\end{array}$ & India \\
\hline Yasuda (2011) & Benoue National Park & $\|$ & 1968 & 1968 & 40 & 2004, 2009 & Private: for profit & Cameroon \\
\hline
\end{tabular}




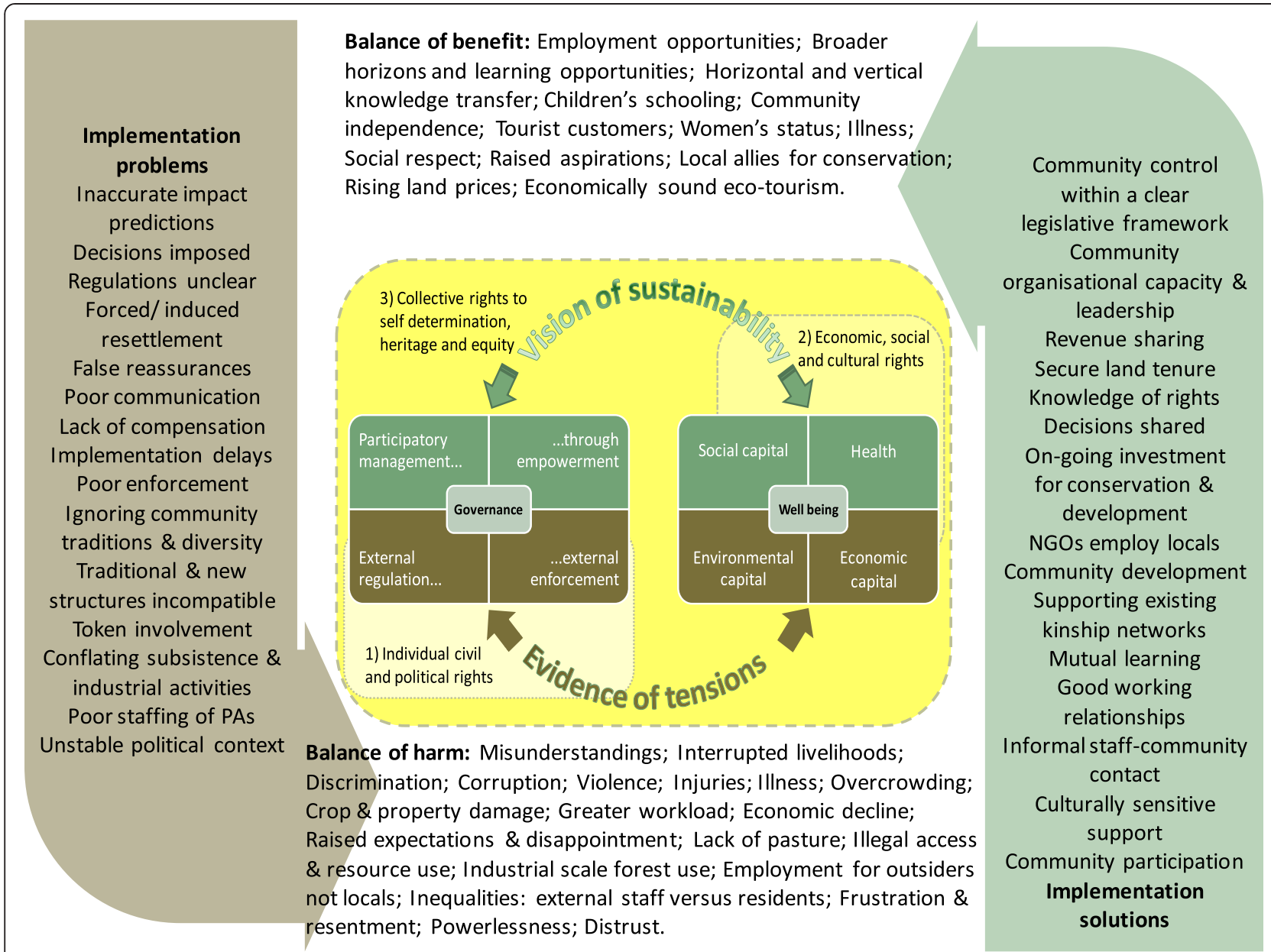

Figure 11 Summary findings from the qualitative synthesis.

managing the area prior to its official status, should play an important role in management [24].

The second source of tension is the lack of clarity in regulations and maps pertaining to protected areas. Mehring et al. [26] investigated regulatory institutions in two villages in an Indonesian park established in 1982. In one, new regulations about forest land and products drawn up by the mayor and customary organisation were neither written down, nor completely implemented. There was support for state zoning of the Park to allow traditional access to the forest for local people, but disagreement about the zone boundaries. Effective village sanctions were considered important, but confusion about when to apply them appropriately arose from discrepancies between state rules and local institutions. In Cameroon, 'traditional hunting' was still allowed in 2001 the territories outside protected areas (IUCN IV) so long as the products were for personal use, and not sold [30]. However, whether 'traditional' hunting referred to the people involved, the weapons employed, or some other characteristic was not clear. Only allowing 'traditional' weapons, depending on the definition, might outlaw common traditional practices such as the use of snares (metal wire), arrows (steel tipped) or rifles. The ban and uncertainty surrounding poorly defined traditional hunting led to tension and mistrust between locals and conservation agents. In Uganda, the legal agreement protecting the Mount Elgon National Park, established in 1951, was flawed as it failed to refer accurately to maps or related by-laws, statutes or other documents [31].

Where regulations precluded living within an area, resettlement could be forced or induced. In Cameroon, forced migration and a violent confrontation prompted villagers to accept resettlement outside familiar territories, against the recommendations of earlier research [29]. Enacting laws to drive resettlement resulted in an integrated conservation and development plan that failed, leaving villagers bitter and sceptical. Expulsion to make way for the privately managed Moremi Game Reserve in Botswana included huts being burnt down as residents got loaded into trucks for relocation outside the reserve [32]. Residents were forced to relocate (for a second time) 
by the suspension of the provision of all social services such as water supply, health facilities, shops, schools and communication services.

Although labelled as 'voluntary', because the term 'involuntary' is politically problematic nationally and amongst international donors, the resettlement from Limpopo National Park was widely recognised as 'induced' by planning blight and economic decline [33]. Although consulted about resettlement, residents' views were then disregarded.

"Since the park was made we were supposed to leave. Since they said that, people don't construct houses, we don't plant trees. This house was built in 2000 but it was never really finished because the park came.

There were trees but we stopped planting and the old ones died. [papaya]. No one is investing, not to do things for nothing. Even now that we have accepted to leave, the park does nothing" (p443).

Inadequate or non-existent compensation was a concern expressed in many studies; for loss of property or land in 1960s [34], for access or use restrictions in India in 2007 [35], for environmental protection by owners of Swedish forests (IUCN Ib and II) in 2001 [36], for resettlement in 2001 or for loss of crops or livestock in 2007/8 Mozambique (IUCN II) [33], for personal injury or property damage from wildlife in South Africa (IUCN II) in 2001 [37], and in Tanzania (IUCN IV) where there was no compensation policy at the time of the study in 1996 [38]; or for loss of jobs or land in China (IUCN V established 1986) [39]. For this last case, some compensation had been made in the form of new homes, crop seeds, lump sum payments, and subsidized education, electricity, and water fees, but views differed on its nature and adequacy [39]. Residents of the Sariska Tiger Reserve (India, IUCN IV) were generally discouraged by staff from claiming compensation for restrictions to access or use forest products [35]. Some villagers were never told they had a right to compensation. Another, on asking for compensation, one villager was told by a forest officer:

"If you the villagers insist in living in the forest, then be ready to accept as well all the consequences deriving from your choice. You could live elsewhere".

Opposition to the Selous Game Reserve in Tanzania grew amongst those losing crops and livestock to wildlife without compensation [38].

Poor communication between communities and authorities was typical. Residents of the Dja wildlife reserve in Cameroon (IUCN IV, established 1951) reported being "neither informed of nor invited to participate" (p.208) in their village becoming part of a protected area but informed later (without being able to give a precise date) of the existence of a conservation initiative by the authorities [30].

Such problems are not restricted to developing countries. In Norway, several residents near a IUCN II category area found the process one-sided and undemocratic because national interests took precedence over local knowledge [24]. Petrzelka and Marquart-Pyatt [27] describe the growing anger of residents and their diminishing trust in agencies to make good decisions about the management of the land after the Grand Staircase Escalante National Monument was established in Utah, USA, in 1996 with no prior consultation or publicity. Trust diminished further over the next ten years later as residents saw roads closed and cattlemen's leases rescinded, despite prior reassurances to the contrary. Restrictions on visiting the park stoked anger amongst residents who felt locked out of our backyard', saying that 'law enforcement is gun-toting like we're a bunch of criminals'.

Even with management of Kenyan national reserves being delegated to a local level, and rangers and wardens claiming to initiate and maintain dialogue, residents are disappointed with the processes of communication [40]. Most of the protected area staff considered their informal word of mouth network sufficient for communicating with local communities about important management decisions.

"Of course we cannot conserve this wildlife without the help of these communities. There must be that, a good relationship between the park and the community. So we normally go to the [homes and villages], we have meetings with them, tell them that these resources are also theirs, these are their resources." (Park ranger, p55)

In contrast, most community members thought that communication between the reserves and communities was limited or non-existent, where decisions were made without opportunities to provide input or ask questions.

In addition to the poor communication between authorities and residents, were the difficulties encountered with communication between residents. The Qwaqwa National Park (IUCN IV), South Africa had been established for the purpose of ecotourism; however, this did not suit the livelihoods of stockholding families and others would have preferred the land to be subdivided for agriculture. Amongst all this disagreement, some residents were more able than others to make their voices heard, and family conflicts escalated as housing became more crowded because erecting new homes within the park was forbidden [41]. In Lore Lindu National Park, Indonesia, the weak point for communication was between villagers and their leaders [26]. Although the 
village leadership was active in the negotiations about the park regulations many ordinary villagers had never heard of the agreements.

In Australia, at the Purnululu National Park (IUCN II) relationships between different indigenous groups were so acrimonious that one group withdrew from the management of the park [22]. In Slovakia, local authorities near Slovensky Raj Park (IUCN II) tried to make management of the park a focus for building relationships and developing mutual trust between different groups [42]. Communication problems could be compounded by new regulatory arrangements being incompatible with traditional ways. For instance, very few Mexican farmers applied for resource use permits because the formal biosphere (IUCN VI) rules competed with customary rules [28]. The formal rules were generic and did not take into account local variation in natural resource management. These mismatches created feelings of frustration:

"The reserve is like a beautiful woman whom you cannot touch. It does not do you any good. The hills are rich, but a poor man stays poor" [28] p205.

Contrary to tradition, only people living on the borders of Mount Elgon National Park (IUCN II) in Uganda were given rights of access [31]. The new outsiders were required to pay the 'insiders' for access, even though half the insiders thought outsiders should have equal rights of access.

Even where access was allowed, as in Permululu National Park, Australia, costs of transportation across long distances over rough ground could be prohibitive [22].

In Indonesia Mehring et al. [26] attributed some of the difficulties of incompatibility to the government's indifference to cultural and social diversities when managing the Lore Lindu National Park (IUCN II). Indigenous people respected their own traditional informal rules that suited traditional use rights and sanctions at the village level. Elsewhere, more prosperous and ethnically diverse villagers, growing more cash crops, referred not to traditional institutions but to economic power structures, where there was a widely spread laissez-faire attitude to resource use. With forest resources and agricultural land in short supply, villagers had no alternative to using the Park to extend their land. The State's formal rules interacted with traditional informal rules, leading to confusion and conflict. Migrants struggled to implement traditional informal rules, and indigenous people failed to obey state-induced laws.

Traditional land ownership rights for indigenous populations were also contested in Australia, both between local residents and protected area management, and amongst local residents of different groups living near Purnululu National Park (IUCN II) [22].

A major challenge to developing and implementing regulations to protect areas is distinguishing subsistence activities for a sustainable environment from larger scale industrial activities. As mentioned above, this challenge was seen in Cameroon where regulations failed to distinguish clearly 'traditional' hunting methods for personal consumption from commercial hunting [30]. In Masoala National park (IUCN II), Madagascar, residents acknowledged that some members of the community benefiting from illegal lemur hunting and timber harvest [43]:

'people [who] wanted easy money, especially the youth, so they went into the park to cut rosewood' [p160].

However, much greater damage was done by industrial scale rosewood logging for international markets, and Park agents have limited legal powers over loggers.

"people from all over come to this area to cut rosewood, there is no other way to get money than from valuable wood" (Park resident p.160).

'[international] demand is driving the outside buyers of rosewood, and this is a much bigger issue than lemur hunting.' (Park Manager, p.162).

Similarly in Cameroon, the impact of residents hunting in Dja Wildlife Reserve (IUCN IV) to ensure a diet that includes animal protein is minor compared with intensive industrial logging which opened up forest tracks and thereby provided access for well organised, commercial poachers to use the tracks for transporting their game to city markets [30] p.208.

Ironically, it was a combined forestry management and community development project in Ecuador that opened the eyes of indigenous people to the potential benefits of logging; when profits were not what they had hoped for, they started making deals outside the community with industrial loggers [44].

Implementing regulations that have disadvantages for local communities is challenging enough. The relationship between residents and park officials in Masoala National park officials in Madagascar (IUCN II) was further damaged by absenteeism amongst staff who, unlike many locals, had the privilege of employment yet lacked training and clear job expectations, and had little interaction with residents [43].

Implementation solutions Blunt regulations imposed by external authorities have been widely disregarded so that protected areas have continued to be exploited on domestic and industrial scales. Studies have focused on efforts to improve communication, draw on indigenous knowledge and share decisions to combine community development with environmental conservation. They have had mixed success. 
The Lore Lindu area in Indonesia was established as a UNESCO Biosphere reserve in 1977 and a national park (IUCN II) in 1993. Since then participatory approaches have been advocated for managing Biospheres [45] and protected areas more widely [46]. Initial efforts to impose external regulations failed and in the late 1990s, the park authority, NGOs and village representatives began to negotiate Community Conservation Agreements [26]. Within designated zones, village conservation councils were the bridge between the Park authority and the community for planning, implementing, evaluating and reporting the results of the Agreement. Despite the village leadership being active in the negotiations, communication between the Park authorities and the whole community was poor, so many ordinary villagers had never heard of the agreements. The Agreements covered use of forest products and land and the village conservation councils were responsible for monitoring activities. The council could employ punishments or sanctions, which were usually based on village traditional rules. Insights into this system came from NGO interviewees. A collaborative management approach aimed to minimise the gap between the park management and the people, through participation of local inhabitants and integration of local rules. Respect for the rules was greater where they were 'more practical' having been locally adapted, and allowed incomegenerating possibilities.

In Mount Elgon National Park, Uganda (IUCN II), as in other African countries, a similar 'fortress management' or 'fence and fine policy', based on systematic evictions, exclusions and prohibition of using natural resources, met increasing resistance [31]. Lack of success with 'fence and fine' policies prompted approaches with greater participation of local people in management and changes in regulations to legitimise sustainable use. Establishing the agreements was difficult even with guidelines and training for park staff; converting staff from law enforcers to community collaborative workers was difficult. Nevertheless, meeting locals and getting to know them improved relations. Some local people acknowledged that their initial reluctance lessened as they met staff and learnt more about the resource base; while a third of respondents did not participate at all.

Once established, the agreement provided greater clarity over rights and duties, and opportunities for long term planning about livelihood strategies. However, as a legal document, the agreement was flawed as it failed to refer accurately to maps or related by-laws, statutes or other documents [31]. Subsequently people were more positive towards the park, its resources and staff. However, as the focus was on the park rather than the community, people were sometimes organised according to what resources they collected, rather than by other socially relevant criteria such as ethnicity, kinship, location, wealth etc. Contrary to tradition, only people living on the park borders were given rights of access. The new outsiders were required to pay the 'insiders' for access, even though half the insiders thought outsiders should have equal rights of access. Conflicts arose from this situation and threatened the agreement's endurance. In such sensitive situations, staff need the socio-cultural skills to understand, interpret and interact with local people about livelihoods, conflicts and challenges in appropriate ways. Reports of misuse and corruption remained common. Nevertheless, collaborative arrangements improved relations and benefited biodiversity and livelihoods.

Sletten's findings in Mount Elgon Park (IUCN II) are supported by other studies. Elsewhere in Uganda supporting community's transition from a hunter gatherer to a settled farming community in a culturally sensitive way was more likely to result in community satisfaction and personal efficacy [44]. Training and capacity building by charities and NGOs led to an increase in skills and knowledge and new income generating activities. Two NGOs working with local people helped to organise efforts around existing kinship networks and this community reported the highest states of economic development compared to other communities. At the other end of the scale these communities were willing to sacrifice their land claims to join relatives in other areas and access charitable projects there leaving the settlements struggling to maintain a viable community.

In Masoala National Park (IUCN II), Madagascar, residents who were more familiar with Park staff viewed the staff as well as the Park more favourably than residents who were unaware of staff or who had had negative interactions with Park agents [43]. Residents were confused by the different NGOs' responsibilities and changing priorities. A park manager and a local town official both considered community development as essential for maintaining a protected area. There was local support for protecting the park by providing community benefits through alternative livelihoods. However, it is unclear from this whether the benefits essential for behaviour change were the intangible empowerment benefits of community development, or the material benefits.

In Selous Conservation Programme (IUCN category IV), Tanzania, support from communities was greatest in areas where education and mobilisation campaigns had been conducted and benefits were beginning to be derived; findings suggest that the majority of villagers supported the project. The evidence showed that they were motivated to join the conservation programme by promises of socioeconomic benefits [38].

The arrival of western donors and NGOs in Caohai Nature Reserve (IUCN V), China, in 1993 changed the focus from enforcement of resource regulations towards small-scale community development and outreach 
programmes [47]. These included small grants and a micro-credit programme for farmers to start up microenterprises in the hope that they would be less reliant on the reserve's natural resources, infrastructure development, environmental education, a community based natural resource management programme, and school fees for girls from poor families. This involved two employees who had extensive prior experience of working with farmers, and required extensive training in community development, gender issues and a variety of participatory methodologies. The result was many fewer hostile confrontations between local people and nature reserve managers, the participation of local people in conservation activities and farmers contrasting the nature reserve's concern for local people with the indifference of corruption of other government agencies. Farmers now work cooperatively with the reserve to seek resolutions to their own problems, sometimes taking the initiative to raise issues about road construction, sanitation improvements, and agro-forestry projects. The transformation from conflict to cooperation has been dependent on funds from NGOs and donors, which raises questions about the project's sustainability.

Another successful example of cooperative management was on the margins of a category II park in Mozambique where land values increased exponentially. With the support of an NGO residents thrived, benefitting materially from land titles, revenues and empowered by the process of acquiring land titles and setting boundaries [48].

Participatory approaches to governance were not always successful. Almudi and Berkes [49] investigated the relationship between a local fishing community and officials responsible for the creation and maintenance of Brazil's Peixe Lagoon National Park. They took a particular interest in the factors that could empower local fishers to 'defend their rights to remain physically within the park and politically in the conservation policy process' (p.220). The authors also found that fisher communities struggled to participate in discussions essential to securing their 'long-term access to the resources for their livelihoods or to trigger the development of a PA co-management arrangement' (p.225). The following quotes were provided as examples of the fishers experiences:

The authors summarised two of the main barriers contributing to the fishers' lack of empowerment as: weak assistance for developing community organisational capacity and leadership; and lack of basic knowledge on laws and fisher rights.

\section{Well-being}

Environmental (natural) capital

- Appreciation of protected areas other than for economic benefit was found in IUCN categories II,
IV and VI; and both before and after the Durban Accord

- Communities expressed a tension between appreciating the environment and wanting to protect it, and also needing to make immediate use of land or natural products

- They could be encouraged to participate in further conservation measures where they could anticipate socio-economic benefits

Evidence synthesised from seven studies

$[23,25,26,28,29,38,43]$

Even where residents have recognised that conserving a park (IUCN II) and its wildlife is valuable on an individual, local, national and global scale for economic, educational, recreational, aesthetic and environmental reasons, and for future generations, they still lament the economic limitations imposed by restrictions on access, extracting resources and grazing, and the dangers of wild animals [23]. Indeed, some communities refuted the need for resettlement, having managed the land (IUCN II) for centuries; this was confirmed by the authors' historical analysis and portrayal of a pristine forest whose protection was incompatible with indigenous residents as a scientific myth [29].

A range of Mexican farming styles have developed along the spectrum of reciprocal relationships between man and nature (co-production) between wilderness and urbanisation, with farmers valuing the land for its provision of food, water, wood and other products [28]. Despite appreciating protected areas for their aesthetic, environmental products, economic opportunities and spiritual values $[25,28]$ their positive attitudes were lost when conservation regulations (IUCN IV) competed with productive activities such as cattle ranching or growing imported varieties of fruit, or with personal safety [25].

Responses to plans for balancing conservation and economic development appear to vary depending on where the benefits might be felt. For some, it was not an interest in conserving wildlife that motivated participation in a Community-based Wildlife Management Programme (IUCN IV) but promises of socioeconomic benefits to themselves [38]. Whereas other respondents were critical of a programme for promoting economic activities alongside maintaining ecosystems; here tourism was expected to benefit entrepreneurs and rich family owners of coastal lands with tourism potential [25].

\section{Access to land}

- PAs are important to communities for grazing, agriculture, hunting, foraging and spiritual homes

- Relocation and loss of control over land and resources can result in resentment, poaching and antagonism 
- Participating in the process of setting boundaries and securing land rights can be empowering Evidence synthesised from nine studies $[30,33,34,40,41,44,48,50,51]$ from IUCN categories II after the Durban Accord, and from categories V and IV before the Durban Accord

Access restrictions to protected areas (IUCN II) had implications for grazing cattle, hunting and collecting natural products $[33,40,51]$. Local people would like grazing rights in the park (IUCN II), especially during drought, and the opportunity to visit traditional areas and burial areas [51]. Where staff were mostly concerned about illegal grazing; they would commonly impound livestock, and fine owners or refer them to a local judicial ward; in contrast most community members felt the harsh environment justified grazing cattle in protected areas [40]. Communities considered that threats to their environmental capital outweighed any potential economic benefits of living near the national park [50]. Outside the Park residents felt they would not have access to resources so far essential to their livelihoods: access to agricultural land, forest resources and grazing land [33]. Wealthy cattle owners were even less disposed to moving because cattle would need to compete for food and water with host villages, and cattle theft was more common outside of the park [33].

Access restrictions posed similar problems for communities in or near PAs categorised as IUCN IV. Accessing the forest was important in order to obtain resources to support local people's livelihood and for feelings about the forest as 'theirs' [30]. Authors described relocation and loss of control over land and resources resulting in resentment, poaching and antagonism [34] and overcrowding resulted from restrictions on building new homes [41].

More positive views were expressed where land values had increased exponentially on a park's margins (IUCN II). Here residents thrived, benefitting materially from land titles and revenues and felt empowered by the process of acquiring land titles and setting boundaries [48].

\section{Resource use}

- Residents appreciated protected areas for their rich products

- Resource use was common even where illegal Evidence synthesised from seven studies $[28,30,35,39,43,44,52]$ mostly conducted before the Durban Accord and spanning IUCN categories II, V and VI.

Residents viewed protected areas as rich sources of food and other products $[28,30,39,43,44,52]$. They acknowledged that illegal resource use continued despite bans $[39,43,52]$. Some park residents could not conceive of a balanced diet without animal protein and protected areas were still seen as the ideal place to carry out hunting throughout the year [30]. Where dependence on access to products was high, collection continued despite a ban as compensation was not always considered adequate [39].

Once introduced to community based management to harvest, process and sell timber, Ecuadorian communities who were disappointed by high start up costs and slow, small gains made deals with external commercial loggers to raise their profits [44]. In contrast, in Mexico, where conservation is widely valued, natural products were used in a sustainable way [28].

\section{Economic capital}

- Before the Durban Accord (IUCN Ib and II), concerns focused on: reduced employment and tax revenues; reliance on foreign aid without understanding its link with conservation; and unrealistic expectations of the economic benefits of tourism.

- After the Durban Accord (IUCN II), concerns focused on: the meager benefits of tourism; what benefits there were not being shared equitably; with indigenous groups or those less amenable to falling in line with new regulations missing out.

- Concerns about lack of compensation were expressed before and after the Durban Accord about IUCN categories II and IV, and about IUCN V before the Durban Accord. Conversely, in developed countries there was a growing welfare dependency. Evidence of economic capital found in thirteen studies [23,29,34,36-41,53-56],

Before the Durban Accord, the impact of IUCN II parks on the wealth of whole areas was seen in Scandinavia, South America and Asia. Forest workers in Sweden associated environmental protection with lower levels of employment and production in commercial forestry [36]. Politicians anticipated conservation policies leading to lower tax revenue and greater emigration [55]. In Nepal communities were developing a dependence on foreign aid, with some people considering it as an expected source of income and not necessarily associating its benefits accrued with conservation efforts [23].

Some people living in or near national parks (IUCN II) were concerned about neighbours having unrealistic expectations of the economic benefits derived from tourism and eco-lodges [55]. Others were unaware that community development was one of a park's primary objectives only initially [43].

Benefits of tourism to national parks (IUCN II) were seen as meagre, and distribution of revenues from protected areas was considered inequitable or of little benefit 
to indigenous communities [40]. Park and eco-lodge staff tend to be wealthier than community residents [43,55]; and local people felt overlooked for employment, with opportunities favouring neighbouring communities and those deemed amenable to new regulations [29]. Similar concerns about few or unevenly shared benefits and opportunities were expressed in other, IUCN IV, protected areas $[34,41]$.

Communities across IUCN categories, before and after the Durban Accord, often considered as inadequate the monetary or in kind compensation available for: forestry constraint; resettlement; loss of land, crops, livestock or jobs; or personal injury or property damage [33-39].

Environmental protection is associated with economic decline in high income countries. In Utah, USA, local residents saw more tourism but no economic growth as a result of establishing a protected area [27]. In Slovakia, although residents living near Slovensky Raj National park (IUCN II) anticipated multifunctional forest management as a source of employment and income generation, in practice the socioeconomic situation worsened, particularly for minority ethnic communities, with reduced employment and changes in welfare support [42]. With their lack of experience and opportunities for involvement in small businesses and local or regional planning, minority ethnic groups saw no viable economic options.

The most positive findings about social and material benefits came from an NGO funded study with one academic author and one author employed by an ecolodge, although they too shared concerns expressed above [55].

\section{Social capital}

- Changes in livelihood strategies have influenced the shape of households and the strength of social ties, and introduced new inequalities within communities.

- PAs have been established in areas inhabited by various ethnic groups. The pressures resulting from regulatory and economic changes have introduced tensions or exacerbated historical tensions between them. Evidence about social capital in PAs was found in eight studies [30,35,38,39,41,44,55]

Slater [41] noted that households configured themselves in a way so as to maximize livelihood diversification; sometimes to the detriment of familial relationships. Households could be separated geographically by livelihoods, or overcrowded because sharing dwellings allowed younger adults to rely on the support of older adults claiming pensions.

Residents saw a direct link between livelihood diversification and changes in cultural traditions and traditional relationships amongst local people; both positive and negative $[44,55]$. Making the transition to a settled agrarian life for some meant a decrease in the traditional communal work ethic and less frequent use of their native language [44,55]. Stronza and Gordillo [55] saw changes in social ties within communities when some began working in the tourism. Locals who secured employment in eco-lodges (IUCN II) talked about their work limiting participation in gatherings traditionally employed to complete community tasks, and how communities tended to charge eco-lodge employees more because they were richer - it was buying them out of their community responsibilities. Working in eco-lodges opened villagers' eyes to new opportunities and a wider social circle but this was at the cost of leaving their family and community. More direct commitment to conservation in a national park (IUCN II) also caused family pressures where the Village Scout scheme took young men away from their family and farming responsibilities [38]. Elsewhere (IUCN V), investment in communities was mentioned by park staff and by residents; this included the aim of establishing a special university training program to prepare students to fill key park management positions [39].

Villagers emphasised the importance of social relations as part of their survival strategy and expressed apprehension about the weakening and possible disappearance of these linkages as a result of displacement. These relations were particular important in times of struggle such as periods of drought [35 p.60]. Stronza and Gordillo [55] considered that communities rich in social capital may not only be better able to manage changes associated with ecotourism, but that such changes in social capital can collectively sustain local institutions, which may subsequently be critical of conservation efforts. In contrast, the community managed forests in Ecuador created new business relationships and improved the community's social standing with other indigenous groups in the area [44].

Although ethnic identities could strengthen social capital within groups, they more often created tensions between groups. Some ethnic groups were resentful as they perceived others receiving community development preferentially, even though the authors saw these community development efforts as culturally inappropriate [44]. As noted above, migrants struggled to implement traditional informal rules, and indigenous people failed to obey state-induced laws. Some of these difficulties were attributed to the government's indifference to cultural and social diversities when managing the Park [26]. At the level of implementing regulations, residents objected to the leniency of guards towards those who were wealthier or ethnically related [23].

\section{Health}

- New diseases associated with changes in lifestyles were attributed to forest evictions or changing from a nomadic to sedentary existence. 
- Accidents and injuries resulted from conflicts between guards and residents; animal conflicts resulted in increased workload and exhaustion as well as injuries

- Sexual aggression was more common when women were less protected following necessary changes in working patterns or kinship ties.

Evidence of health and protected areas was found in five studies $[35,38,41,44,54]$

In one study, community members evicted from forest unanimously spoke of their exposure to new disease when integration with other groups began; authors confirmed that the community, particularly children, were seriously affected by malaria which did not exist in the forest, and that HIV/AIDS is also appearing [44]. Their forced transition to an agrarian society has cut them off from their access to and knowledge of traditional medicinal plants they previously used to stay healthy. Elsewhere, villagers reported the lack of access to basic health services and Torri [35] confirmed that child mortality was high in isolated forest villages, where common illnesses, easily treatable given basic medical facilities, could lead to death.

Residents described how crop raiding by elephants lead to food shortages and greater workloads, especially amongst women who suffered more from insect-borne diseases and heat exhaustion. When elephants had damaged water pipes, women risked drowning when collecting water from unsafe sources [54]. First Peoples [44] reported women's safety being compromised as men worked further afield, and as women were drawn out of their homes for new roles and that the erosion of kinship ties maybe reducing protection from male sexual aggression [44].

\section{Inequalities}

- PAs and residents' responses to them have exacerbated existing local ethnic tensions

- Participation in PA governance has favoured people already advantaged by their socio-economic position

- PAs impact unequally on people depending their socio-economic position, such as the size of their business, their legal land tenure or their gender

- New tourism enterprises have tended to employ outsiders rather than locals

Evidence of inequalities arising from PAs was found in 15 studies [23,26,31-33,35,36,38,41,44,50,51,54-56]

People living in and near PAs differ in many ways. Some indigenous people living near each other come from different ethnic groups. Some PAs include indigenous communities, new immigrant communities and residents long established following historical migrations. Individuals differ in terms of their occupations (e.g. subsistence farmers, day labourers, tourism employees), tenure (landowner or not), gender, education and wealth.

Differences in wealth accrued from ownership of land or livestock have been influenced by wildlife conflict $[38,54]$ and by PA regulations about land access or livestock numbers, including whether or not people complied [41]. Smaller businesses and entrepreneurs less able to control shocks may bear the brunt of further restrictions on the forest products [36].

Some of these individual differences have arisen at least in part from inequalities between ethnic groups or from discrimination between indigenous groups. For instance, some indigenous people have discouraged immigration and excluded immigrants from community governance procedures [26]. Within and between ethnic groups, people have taken advantage of others who earn more from the presence of the PA [55] or from people struggling to make a living who sell land legitimately owned only to claim protected land illegally [26].

Misunderstandings or prejudices about the histories or abilities of communities led authorities to pursue decisions which those communities found damaging [32,33]. Authorities also discriminated within communities by exercising policies that protected landowners but not other longstanding residents [41]; or by involving people chosen for their age, wealth, education and position rather than practical relevant knowledge [31]. Ethnic discrimination has been introduced by PA legislation that forbad resource use by indigenous people but allowed resource use by predominantly white landowners [51]. Where ethnic discrimination predates establishment of PAs, historic practices of favouritism have strengthened as land has become more valuable and rare because much of it has been put aside for conservation [50]. Prejudice and nepotism have been the source of favouritism or corruption amongst PA employees [23].

Some differences have arisen from commercial or conservation enterprises providing more earning power for some, but not all, roles [56]. Some eco-tourism enterprises have a history of being poor employers of local casual labour [32]. Financial inequalities were introduced when spent funds resulted in financial support for some but not others, and bank loans have been refused on the grounds of having an address in a protected area [44].

Women were vulnerable to attack and injury from men or wildlife $[38,44,54]$. Female heads of households were vulnerable financially as they tried to balance paid labour with subsistence farming [41]. More may have been learnt if some women had not been reluctant to express their opinions to researchers [35].

\section{Narrative synthesis of quantitative evidence}

In this section we provide a narrative overview of all included studies providing quantitative data on impacts. 
The studies are divided into six subsections; produced iteratively based on the outcomes reported in the accepted literature (see typology in Figure 12). We do not claim that these divisions are definitive or optimal but they do provide a pragmatic breakdown of a complex body of evidence. A summary of data presented in the included studies is provided as Additional file 7. A more detailed set of data extraction tables is available as supplementary material. Of the 79 studies included in this synthesis 63 were categorized as having 'high', 11 'medium' and 3 'low' susceptibility to bias. Additional file 10: Table S1 displays the 14 studies and 33 outcome measures that were categorised as having low and medium susceptibility to bias. Additional file 11 provides detail of critical appraisal and the basis for assigning susceptibility to bias for all 79 studies. In the following text we concentrate on results reported in these 14 studies. Here where we discuss studies' 'susceptibility to bias' we refer to their categorisation resulting from our critical appraisal. In some instances we also identify specific types of bias (defined in Table 5, below) and in others we refer to shortcomings of the study design and implementation that implicitly increase the studies' susceptibility to bias.

It is important to note that since the majority of studies were identified as being highly susceptible to bias, the results of these studies are not considered further, either individually or in general. These studies are unreliable both alone and in concert. Because this group of potentially biased studies is unreliable, we cannot summarise their findings any more than we can discuss individual results. However, for completeness we also consider the diversity of outcomes reported by all studies. Furthermore, we avoid vote-counting, where the sum of all negative, positive and neutral study results are calculated. Vote-counting is unreliable because it assumes that a significant finding is evidence that an effect is present and a non-significant finding is evidence that an effect is absent. This former statement is true, but the latter is not (see Borenstein et al. [21] for further details of vote-counting).

\section{Ecosystem goods}

- 17 studies identified

- Only two studies not highly susceptible to bias ('medium' susceptibility to bias)

- Nyahongo et al. [57] found that meat and fish consumption increased with proximity to Serengeti National Park

- Sarker and Røskaft [58] found residents' perception of timber and fuelwood benefits from four PAs in Bangladesh decreased with distance from the PA boundary

- High susceptibility to bias in remaining studies related to low methodological detail, confounding variables, and weak experimental design

Of the 17 studies reporting results on ecosystem goods, only two studies had designs that were not highly susceptible to bias (i.e. medium). Nyahongo et al. [57] reported the number of meat and fish meals consumed by survey respondents to be significantly negatively

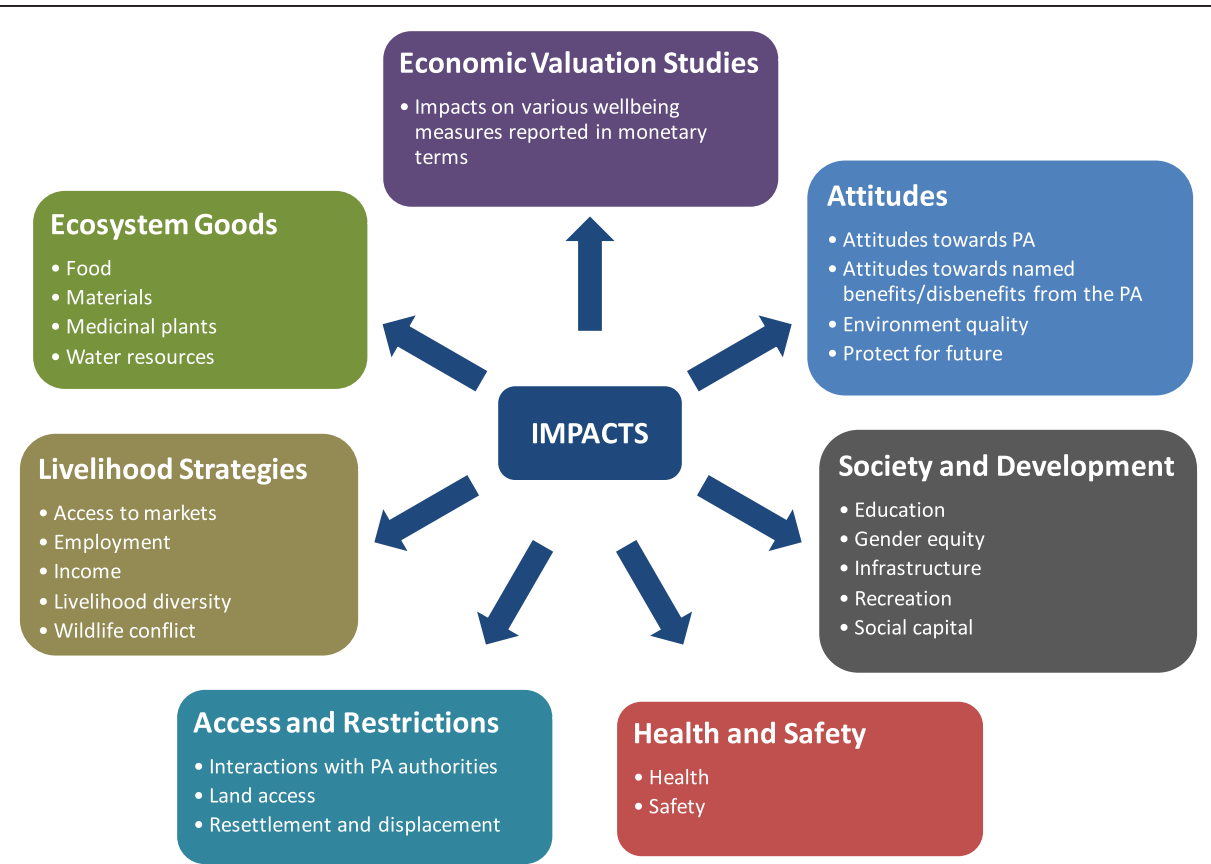

Figure 12 Typology for structuring the quantitative synthesis of impacts of protected areas. 
Table 5 Definitions of bias mentioned in the narrative synthesis

Recall bias
Social desirability bias
Questioning bias
Neyman bias
Attrition bias
Optimism bias
Hypothetical bias
Strategic bias

Imperfect recollection of past events by respondents. Generally worsened by longer periods of recall

Tendency to respond to questioning in such as way as to be viewed favourably by others

Questioner leading respondents to reply to questioning in a certain direction

Arises from a time lag between exposure and sampling such that undetected drop-out of participants may occur before the study begins.

A skew in results where participants are lost between measurements at two time points (potentially as a result of the exposure) during the study

A belief by a respondent that they are less likely to experience a negative event relative to other respondents, or over-optimism on the part of analysts or interviewers, about the effects of a project.

Failure of respondents to consider the true budget constraints in responding to financial questioning Tendency for respondents to alter their answers in an attempt to influence an event correlated with distance from Serengeti National Park boundary. The authors' statistics account for a range of other potentially influential variables, contributing to the studies favourable susceptibility to bias rating (medium). The study's results indicate that meat consumption increased with proximity to the park at a rate of 0.218 $( \pm 0.052)$ meals per week per $\mathrm{km}$, whilst fish consumption increased at a rate of $0.931( \pm 0.205)$ meals per week per $\mathrm{km}$ (assuming units in analyses are identical to units described in the methodology). Sarker and Røskaft [58] found that people inhabiting the areas surrounding four protected areas in Bangladesh identified more benefits from the protected area in the form of timber and fuelwood extraction closer to the park boundaries than further away, with an associated odds ratio of 1,000; i.e. "The odds that respondents living closer to the protected area boundary reported a higher level of benefit from timber and firewood because of the conservation programme were 1,000 times greater than those for respondents living further away".

Thirteen studies used questionnaires and semi-structured interviews for data collection, resulting in a higher susceptibility to bias since reporting by the respondents can be subject to recall or social desirability bias. Fourteen studies reported 39 different (but not all independent) outcomes related to food and materials, comprising a mixture of foods obtained by hunting and gathering, agriculture and purchased, as well as other indicators such as land area under cultivation or grazing, availability of fodder, support for agricultural development, dietary diversity, gathered fuelwood and other plant products including timber, and also change in ownership of goods. Five studies reported seven different (but not all independent) outcomes related to water resources: three studies related predominantly to water quality (e.g. households relying on least safe water resources) and two to water availability and supply. Only one study reported one outcome related to medicinal plants/ animals.

Studies reporting data on common themes differed significantly in the precise outcomes measured. For example, whilst Bajracharya et al. [59], Mehta and Kellert [60], Naughton-Treves et al. [61], Rinzin et al. [62], and Okello et al. [63] all measured accessibility and quality of water resources, there was insufficient conformity to allow meaningful synthesis.

Other studies could not be used to infer reliably any effect of a protected area for a range of reasons, including; (i) they recorded change over time with no spatial comparison and no adequate basis for determining whether the changes observed were attributable to the effect of the protected area; (ii) they made a spatial comparison, but location relative to the protected area is clearly confounded with a range of other important site variables; (iii) the scale of the spatial comparison was too small to use it to infer effects of the protected area on the specific outcomes recorded in the study; (iv) time elapsed between the creation of the protected area and the study was too short to infer any effect of the protected area.

\section{Livelihood strategies}

- 43 studies identified

- Eight studies not highly susceptible to bias (1 'low' and 7 'medium' susceptibility to bias)

- Four studies report poverty-related outcomes, finding beneficial impacts of land protection in all cases

- Wildlife conflict is relatively well-studied (18 studies and 47 outcomes; 5 studies with 7 outcomes 'medium' susceptibility to bias. The majority reported significantly more problems with proximity to protected areas than further afield

- Lundgren [64] found no significant difference in income growth or forestry/tourism sector employment as a result of protected areas in Sweden

- Household income [65] and consumption [66] were found to increase with proximity to/within protected areas

Studies in this set report on access to markets, employment, income, livelihood diversity, human development 
measures and wildlife conflict (Additional file 7). Wildlife conflict was the most frequently reported outcome measure in this group, contributing 18 of the 43 studies and 47 of the 101 outcomes. Only five of these 18 studies were not highly susceptible to bias, all bar one [52] showing significantly greater crop and livestock loss closer to protected areas. Two of these five studies employed questionnaires to elicit perceived disbenefits, whilst three studies used observations of conflict. Lundgren [64] found no significant correlation between protected areas and income growth or employment in either tourism or forestry sectors in Sweden. Sims [66] reports higher consumption in regions with a high 'share' of PAs compared to a low 'share' in Thailand. Richardson et al. [65] found households within game management areas (GMAs) to have greater income across a number of Zambian PAs than those outside GMAs.

Four studies reported poverty-related measures (human development measure outcomes) as either poverty index or poverty headcount. All four studies found significant beneficial impacts of protected areas on poverty alleviation in Costa Rica [67], Thailand [66,67] and Bolivia [68]. The study by Andam et al. [67] involved subsequent in-depth reanalysis in two later publications $[8,69]$. These later analyses showed that along with protection alleviating poverty, poorer areas (measured at baseline) were found to have greatest level of poverty reduction than those that were less poor [8]; and that poverty alleviation was also associated with characteristics that reduced the efficacy of deforestation prevention (i.e. where protection had been assigned to land that was unsuitable for agriculture, near major cities and infrastructure, and where agricultural employment is low) [69].

The remaining studies were judged to have high susceptibility to bias and the following examples are only illustrative of the diversity. Foerster et al. [70] report that purchasing power is lower in villages closer to PAs in Gabon. Cardozo [71] conducted a questionnaire-based site comparison of communities inside and outside AllpahuayoMishara National Reserve, Peru and reported changes in income and livelihood diversity. Annual income from agriculture was lower inside the PA whereas income from domestic animals and palm products was higher. Kayser et al. [72] report greater annual transfer of money to SMEs through contracts financed by Addo Elephant National Park, South Africa. Saayman \& Saayman [73] provide data on self-reported change in the business environment around South African PAs. This provides weak evidence that opportunities for and turnover of business has increased as a result of the PA.

\section{Land access and restrictions}

- Six studies identified
- All studies are highly susceptible to bias due to lack of methodological detail, non-random sample selection, spillover, questioning bias and uncontrolled confounding variables

- The majority of outcomes relate to restrictions on access or extraction

- Two outcomes related to the perception of relationships with park employees

The six studies in this set report on 11 different (but not all independent) outcomes related to land and resource access, interactions with protected area authorities, displacement and resettlement (Additional file 7). All had a high risk of bias in their study design because of a lack of detailed description of their methodologies. In cases where the description was detailed, specific risks of bias were identified; replicates were non-randomly selected $[59,74]$ and spillover, questioning bias, and confounding variables were not accounted for $[59,75,76]$.

\section{Health and safety}

- Nine studies identified

- Only one study identified as not highly susceptible to bias ('medium' susceptibility to bias)

- Korhonen et al. [77] found highly variable infant mortality rates in and around Ramonafana National Park in Madagascar, with slightly higher levels outside than inside the PA, although the pattern is not clear

Studies in this section look at health in the population and access to health services (Additional file 7). Studies reported a limited range of outcomes relating to health and safety, but the reliability of findings in general was compromised due to various aspects of study design which make them highly susceptible to bias, such as a lack of comparator or non-random selection of study sites or participants, or incomplete reporting, with no details about selection of study populations, validity of survey instruments or survey response rate, $[73,78]$. The only study in this group with 'medium' susceptibility to bias, Korhonen et al. [77], report a case-study of reproductive health from a 10-year old Integrated Conservation and Development Project (ICDP). The study was of BACI design comparing purposively selected (for representativeness) villages and municipalities within a $3 \mathrm{~km}$ belt around Ranomafana National Park (the 'peripheral zone') (7 villages, 6 municipalities) with those outside this zone (6 villages, 4 municipalities). Data on modern contraceptives suggest an increase in use over time, a decrease with increasing distance from the park and variability in use within 'park' villages attributed by authors to varying access to ICDP activity, but also to educational status of women, local culture and religious 
beliefs. Fertility levels were estimates rather than direct measures and do not indicate any change over time or differences between park and 'outside' villages. Visits to the health centre for pre-natal care increased over time in line with national trends and did not differ between park and 'outside' village residents. The data presented for infant mortality were drawn from census data pre-1999 and showed year-on-year variation and no clear differences between 'park' and 'outside' residents, although a slightly higher mean over an 8 year period outside than inside the PA. Post-1999 data were health centre data and only related to health centre births and thus may be subject to bias if a higher percentage of more problematic births occurred at the health centre as opposed to in villages.

\section{Society and development}

- 13 studies identified

- Two are not highly susceptible to bias (1 'low' and 1 'medium' susceptibility to bias)

- Sheppard et al. [79] found a greater number of infrastructural developments inside Wechiau Community Hippo Sanctuary in Ghana than outside

- Korhonen et al. [77] found no difference in the percentage of girls in primary schools either over time or inside relative to outside Ramonafana National Park in Madagascar, but a possible slight increase in the percentage of girls in the third grade over time was found inside the PA

Studies in this set include measures of education, empowerment, infrastructure, recreation and social capital. Thirteen studies reported data on 54 development-related outcome measures. Two studies were classed as having low (1) or medium (1) susceptibility to bias. Sheppard et al. [79] found a greater number of developments in various aspects of infrastructure inside Wechiau Community Hippo Sanctuary in Ghana than outside. Korhonen et al. [77] found no difference in the percentage of girls in primary schools over time and no difference inside relative to outside Ramonafana National Park in Madagascar, but they did find a slight increase in the percentage of girls in the third grade over time inside the PA (but not outside).

All remaining studies were judged to be of 'high' susceptibility to bias. There is weak evidence for improvement in education provision following PA establishment in terms of increased number of schools and perceptions of improvement $[62,72,78]$. A questionnaire based on reported change conducted by Saayman and Saayman [73] in multiple South African PAs produced variable responses (in most, but not all, the majority agreed) to statements such as 'participation in community activities has increased', 'the pride that the residents have in their town has improved', 'the opportunities to meet new people has increased'. Other studies report perceived improvements in infrastructure in and around PAs compared to elsewhere or before establishment $[59,78]$ but this is not always the case [62].

\section{Attitudes towards PA and the benefits (or otherwise) they provide}

- 24 studies identified

- Only one study identified as 'medium' susceptibility to bias, all remaining studies are highly susceptible to bias due to lack of methodological detail, confounding variables unaccounted for, and spillover from protected areas into controls

- Sarker \& Røskaft [58] found attitude to PAs to be negatively associated with PA proximity

- Four studies failed to identify the location of 'inside' populations, two studies failed to report the distance of controls from PAs, and in general controls were very close to PA boundaries (e.g. $<1 \mathrm{~km}$ and $2.5 \mathrm{~km}$ )

- Studies reported attitudes towards PAs, attitudes towards identified benefits/disbenefits from the PAs, and perception of environmental change

Studies reporting attitudes were separated into two distinct categories; attitudes towards the protected area or conservation, and attitudes concerning benefits or disbenefits resulting from the protected area. All but one of the 24 studies reporting attitudes fell into the category of 'high susceptibility to bias'. The main issues identified in the critical appraisal were lack of detail in the methods, not accounting for important confounding variables, and, where spatial comparators were used, potential spill-over effects due to the intervention and 'comparator' sites being close together. This latter issue was a particular problem for studies looking at the effect of 'distance from the PA boundary' on attitudes. Thirteen studies examined spatial differences in attitude with respect to distance from the protected area. However four studies failed to report the location of the intervention population inside the protected area. Two studies $[58,80]$ regressed attitude scores against distance from the protected area, but the distances involved were not stated. Control populations in 'inside-outside' studies were generally very close to the protected area boundary, for example $2.5 \mathrm{~km}$ (Sekhar 1998) and < $1 \mathrm{~km}$ [71]; although it was $50 \mathrm{~km}$ in the study of Bonaiuto et al. [81].

Attitudes towards the protected area Fourteen articles reported a wide range of general attitudes towards the PA. Thirteen of these gave respondents' statements (usually a mix of positive and negative statements) and presented data on the percentage agreeing or disagreeing with each statement. One study [81] presented composite 
attitudinal scores made up of responses to a series of questions which were not presented in the article.

In the only study that was judged not to be highly susceptible to bias, Sarker and Røskaft [58] found that respondents from around four parks in Bangladesh had negative attitudes towards the protected areas, and that negative attitudes decreased with distance from each protected area. The remaining studies were of 'high' susceptibility to bias. Bonaiuto et al. [81] reported that regional identity and place attachment were higher inside the Tuscan Archipelago National Park in Italy, but that specific and general attitude scores towards the protected area were lower relative to a control group of respondents $50 \mathrm{~km}$ away. Jim and Wu [82] noted that a higher proportion of people living on the boundary of Shimentai Nature Reserve in China 'disliked' the park than those living $4 \mathrm{~km}$ from its boundary. Finally, Shrestha and Alavalapati [80] observed a positive correlation between positive attitude and distance from Koshi Tappu Wildlife Reserve in Nepal.

In contrast to the above studies which found a positive relationship between distance from the park and attitudes, Cardozo [71], Gubbi et al. [83], Infield and Namara [84] and Sekhar [85] reported higher positive and lower negative attitudes inside protected areas than outside. Other studies found no statistically significant or observable difference in attitudes between inside and outside protected area [86-89] or over time [90].

Attitudes towards named benefits/disbenefits resulting from the park Thirteen studies reported data concerning respondents' attitudes towards named benefits or disbenefits resulting from the protected area. Again, all studies were classed as highly susceptible to bias. Ite [89] found fewer respondents close to Cross River National Park in Nigeria to believe that they have benefited from the protected area than those $5 \mathrm{~km}$ away, although a third group of respondents $7.5 \mathrm{~km}$ from the protected area showed an intermediate perception of benefits. Jim and $\mathrm{Wu}$ [82] reported no significant difference in the perception of benefits from Shimentai Nature Reserve, China, between respondents inside and those $4 \mathrm{~km}$ from the protected area, whilst significantly more respondents inside than outside claimed to have felt losses as a result of the reserve.

Other studies found no evidence that respondents felt that either negative or positive impacts resulted from the protected area $[75,86,88,91,92]$.

A smaller category of studies reported respondents' perceptions of environmental change as a result of the protected area; with respondents in one study predominantly not perceiving a change [90] and two studies reporting the majority to have perceived an increase in environmental 'appearance' [73,93]. However, these studies were of low quality predominantly due to a lack of detailed methodology and shortcomings of the experimental design. For example, Cihar and Stankova [90] lacked a true before-and-after comparison and generated 'before data' by asking informants to recall the past. This is clearly open to recall bias.

\section{Economic valuation studies}

- 10 studies identified

- One study judged as not highly susceptible to bias ('medium')

- Four groups of studies found: cost-benefit analyses; stated preference studies; stated preference combined with a distance comparator; and reported direct financial losses from a PA (e.g. fines/foregone income)

- These studies do not have real comparators (with the exception of the distance comparator): instead they are hypothetical, and as a result are highly susceptible to bias (e.g. optimism bias)

- Studies are too heterogeneous and open to bias to permit meaningful quantitative synthesis of valuations

Studies in this section reported welfare impacts in monetary terms. Economists usually hold that individual well-being is not directly and cardinally measurable, nor comparable between individuals or time periods e.g. [94]. However, changes in an individual's well-being as a result of a PA's existence can be expressed in terms of the amount of money needed to render that individual indifferent to the existence of the PA (the aggregation of such monetary amounts across individuals is common in applied economics, but deeply problematic).

Ten studies were included that estimated well-being impacts of protected areas in monetary terms. Nine of these were categorized as having high susceptibility to bias, whilst one was categorised as medium susceptibility to bias.

Shrestha et al. [95] used a contingent valuation survey with a stratified random sample of 160 households within c. $6 \mathrm{~km}$ of Koshi Tappu Wildlife Reserve, Nepal, to estimate their willingness to accept the PA in terms of foregone resources. They found substantial local onetime costs of 11,776.70 Nepali Rupees per household (1994/1995).

A major reason for excluding economic studies (see Additional file 6) was that they measured the well-being impacts of ecosystems within PAs, but did not isolate the impact of the PA itself. One study [96] carried out a contingent valuation survey of willingness to accept compensation for costs of the protected area's presence on traditional pasture land, with respondents within the protected area and further away. The remaining studies included only hypothetical or 'modelled' comparators. In 
all cases this is done more or less explicitly by the analysts themselves, but many studies also required respondents to mentally construct hypothetical comparators, in order to answer stated preference surveys. Such constructed comparators can be useful and indeed essential when "real" comparators (RCTs, BACI etc.) are unavailable (they may also be used in conjunction with such research designs). However, they are vulnerable to a number of potential biases, such as optimism bias, strategic bias, and hypothetical bias (see Table 5 for definitions).

Without real comparators, direct evidence is lacking on the effects of the PA on individuals. Instead these must be predicted, using whatever information and opinion is available to the analyst or respondent. Numerous assumptions must necessarily be made, and will not always be explicitly stated. This leaves these studies open to wellrecognised biases. For example, cost-benefit analyses are known to suffer from optimism bias, especially when conducted by groups with an interest in the project: in the case of PAs this may be the government or conservationists more generally. For example, the results of Kremen et al. [97] are heavily dependent on optimistic assumptions made about the efficacy of development interventions planned to accompany the PA: no evidence is presented on whether these interventions indeed had the effects assumed by the authors, since the analysis was conducted ex ante.

Stated preference studies are known to suffer from both hypothetical bias and strategic bias on the part of respondents. Hypothetical bias may lead respondents to overstate their willingness to pay (WTP) for goods or services provided by a PA, because they fail to consider their true budget constraint. Respondents may also behave strategically: beneficiaries may overstate their willingness to pay for a PA, in order to increase the likelihood of its establishment if they suspect they will not be required to contribute to it, or understate their WTP if they suspect that this will result in lower user fees. Those who expect to lose from PA establishment may overstate the amount that they would require to receive in compensation for the establishment of the PA (i.e. their willingness to accept (WTA) the PA) to reduce the likelihood of its establishment, or increase compensation payments. Alternatively surveys may under-estimate opportunity costs if the activities concerned are considered sensitive or of dubious legality, and are likely to be under-reported. The results of stated preference studies are also known to be sensitive to the information provided by surveyors, and the precise formulation of the questions. This renders them vulnerable to the same optimism bias noted above.

Methodologically, the included studies fell into three groups: cost-benefit analyses, stated preference studies, and reported direct financial losses from a PA. One study, Kremen et al. [97] carried out an ex ante cost-benefit analysis of the establishment of Masoala NP, Madagascar, disaggregating costs and benefits by local, national and international groups. Both the effects of the PA and the counter-factual (no PA) were modelled, though little detail is presented and the evidence upon which the modelling is based is often rather weak. They estimated that local populations would lose from the establishment of the park, but that this would become a net gain if development projects associated with the park succeeded in raising local incomes. At the national level, there would be a net loss due to the protected area.

Seven studies used stated preference techniques to elicit estimates of welfare gains or losses. Four studies [7,98-100] used contingent valuation to estimate regional or national populations' willingness to pay for existing protected areas in India, Brazil, China and Greece respectively, all four studies indicating generally positive welfare impacts of the PAs on these broad populations. A fifth study, Ascuito et al. [101], similarly estimated local willingness to pay for a fire prevention programme in an existing protected area again finding positive welfare impacts. Two studies $[95,96]$ used contingent valuation to estimate local populations' willingness to accept restrictions on livelihoods imposed by existing protected areas in Ethiopia and Nepal respectively, indicating negative welfare impacts of the PA. In the case of Jemal [96], surveys were carried out with respondents inside and further away from the PA, and found that people within the PA were less willing to accept compensation than those further away, suggesting negative welfare impacts increased with proximity to the PA. Finally, Abbot and Mace [102] present data on fines levied on local people for illegally harvesting fuel-wood in Lake Malawi NP. These fines were levied by the PA but no information is provided on areas outside the PA.

\section{Inequalities}

Assessing the impact of PAs on health or social inequalities would require either individual sound studies with justifiable subgroup analyses, or a set of comparable studies which describe in detail the socioeconomic position of the populations studied $[103,104]$. Neither was available from the extant literature.

\section{Meta-synthesis of qualitative and quantitative evidence}

In attempting to bring together the findings of the qualitative and quantitative reviews it is important to reflect on the differences in their philosophies. The qualitative synthesis is essentially formative and attempts to form a picture of how PAs are perceived to impact on human well-being. As such it can form a template for empirical investigation and hypothesis testing. The synthesis of quantitative evidence is more summative and attempts to test hypotheses of impact. In consequence we should not expect the meta-synthesis to be a simple matching 
of similar studies or outcomes. In this section we summarise the findings of the qualitative synthesis and ask whether the quantitative evidence of impacts can inform the questions raised by these findings or whether it suggests something different.

\section{Governance}

The qualitative synthesis reveals a number of factors that can lead to negative views and impacts of PA establishment: lack of clarity in regulations and boundaries; discrepancies between state rules and local institutions; forced migration, inadequate or non-existent compensation; and poor communication between communities and authorities and government indifference to cultural and social diversities. Negative views on impact of management can arise from poor relationships between residents and park officials. Views on how to lessen negative impacts or achieve positive impacts include: rules that are locally adapted or based on traditional rules; greater clarity over rights and duties; planning focussed on community livelihoods as well as the park; appropriate capacity building; and empowerment through the process of acquiring land titles and setting boundaries. The existence of these views enables hypotheses to be generated on how to achieve change in impact. The synthesis of quantitative measures of impact shows that these hypotheses are yet to be tested. What is absent from the evidence base is a quantitative comparison of costs and benefits to local people of different forms of PA governance.

\section{Well-being}

Environmental (natural) capital The qualitative synthesis presents a range of positive and negative attitudes among local populations towards PAs. Alongside an appreciation and desire to protect the environment were concerns about reliance on those same areas to maintain economic livelihoods. Although the qualitative literature provides evidence of difference views, quantitative evidence to estimate the scale and reach of those views was not extractable since all but one study were highly susceptible to bias,

Access to land The qualitative synthesis revealed two very different scenarios in terms of access to land. The first is resentment at loss of access and the second is benefit from acquisition and value of land on the PA margin. All quantitative studies of impact of PAs on land access and restrictions were highly susceptible to bias and so the current evidence does not allow the magnitude of these scenarios to be assessed.

Resource use A range of positive and negative views were found concerning PAs as a source of natural resources and ecosystem goods. In the synthesis of quantitative evidence of impact only two studies were not highly susceptible to bias, showing that meat and fish consumption was greater in proximity to a PA and that timber and fuelwood benefits were more frequently appreciated nearer another PA.

Economic capital Views expressed on impacts of PAs on economic capital are generally negative, with the exception of some views on the benefits of ecotourism. In contrast the quantitative evidence of impact from three studies on livelihood strategies was neutral to positive in terms of poverty reduction. In particular, there were concerns in Sweden amongst foresters about sustaining employment and amongst politicians about sustaining $\operatorname{tax}$ revenue in the presence of regulations. However, these concerns were not upheld by a quantitative assessment of impact in the same country. All but one of the economic valuation studies suffered from high susceptibility to bias and therefore add limited reliable quantitative evidence to this issue.

Social capital The qualitative synthesis suggests development associated with PAs can exacerbate ethnic tensions through perceived preferential treatment of some communities. There may be a relationship between existing social capital and ability to adapt to new circumstances. Quantitative evidence of impact on social capital is mixed. There is some evidence of positive impact of land protection on poverty alleviation and on housing and infrastructure but also of increasing incidence of wildlife conflict.

Health Views expressed on health of local populations are predominantly negative, including exposure to disease, wildlife conflict and women's safety. Quantitative studies of impact of PAs on health and safety are notable by their absence.

\section{Discussion}

Historical accounts of establishment of PAs provide evidence that substantial negative impacts on local populations can occur and have occurred. Forced displacement of communities is a recurring theme in the narrative concerning negative impacts of PAs e.g. [4]. This review does not seek to question this historical narrative. Community development and infrastructural improvements in close proximity to PAs has also been documented and suggests that PA establishment can be positive (i.e. win-win solutions for biodiversity and human well-being are possible). Establishment of PAs will inevitably lead to impacts on local, and possibly regional, populations but the challenge is to improve our capacity to predict which factors will influence the balance of positive and negative impacts. In this review we have attempted to provide an assessment and characterization of the range of positive and negative impacts in the period following the Rio Summit and establishment of 
the CBD. It was not the objective of this review to revisit this history prior to 1992. The review also attempts to collate evidence on the factors that modify impact, either in a positive or negative direction.

\section{Comparison of qualitative and quantitative evidence}

Although the quantitative evidence is insufficient to draw conclusions about the scale of either positive or negative impacts of protected areas on well-being, it was possible to synthesise understandings from qualitative studies about how positive and negative changes in well-being can arise from establishing and implementing regulations to protect the natural environment, with or without simultaneous investment in community development.

The qualitative synthesis has identified a number of themes in the ways governance of protected areas affect human populations well-being and how PAs are viewed. Some of these themes, such as the impact of land protection on forestry sector employment in Sweden, are reflected in the impacts assessed in studies considered in the quantitative review. Other themes, however, have not been rigorously assessed in the quantitative literature, for example, novel diseases resulting from changes in lifestyle, increased workload and heat exhaustion due to crop raiding, safety risks felt by women as men worked further afield and as women themselves were drawn out of their homes for new roles.

\section{Reasons for heterogeneity}

The identification of variables that influence whether positive or negative impacts will occur would be desirable for supporting decision making on the process of establishment and subsequent management of PAs. Unfortunately the nature of the evidence provides little opportunity to analyse differences in impact among different PAs (see limitations below).

Mode of governance is commonly viewed as a key variable determining impacts of PAs and this is supported by the qualitative synthesis in which many narratives are available on different aspects of governance. However, rigorous tests of governance as an effect modifier are absent. A similar lack of quantitative evidence is apparent for the following questions:

What practices repeatedly lead to negative impacts, and which ones seem to be recurrently improving people's well-being?

Are some of these practices becoming more/less common with time?

Are any costs or benefits associated with particular types of PAs (e.g. size or location)?

\section{Strengths and limitations of the review}

This is the first systematic review of which we are aware that attempts to identify and synthesise, in a transparent manner, the findings from international studies of people's views about the impact of protected areas on their lives. To reduce the likelihood of missed studies, sensitive searches of bibliographic databases were supplemented by other methods to seek out less easily found literature such as unpublished reports from topic relevant websites. Studies providing thick descriptive data spanning the different categories of IUCN protected areas and before and after the Durban Accord offered an excellent source for synthesising understanding about how protected areas impact on people's lives.

During the reading and re-reading of individual studies we found that data often encapsulated the complexity of living in or near protected areas, touching on a multiplicity of interrelated themes. Within the limitation of time and resources for this study we have only been able to present these themes fairly superficially without exploring fully all their interconnections.

The disparate, fragmented literature limits our ability to test the comprehensiveness of the search. In reviewing such a broad and interdisciplinary question it has been a significant challenge to test all the possible sources of relevant material; nor is it simple to measure what proportion of the relevant articles we have been able to access with the time and resources available. Limiting our search to English-language articles may be significant.

Diversity of the literature has limited any assessment of extent of publication bias. Whilst we have attempted to minimise publication bias by employing a systematic search strategy, we have no way of testing for publication bias in the literature we obtained. The selective nature of many studies, in terms of the type of impacts investigated, is also a potential source of bias, since researchers may 'cherry-pick' (possibly inadvertently) those impacts most likely to show a particular effect.

\section{Limitations of the evidence base}

Although the quality of the studies was sufficient to draw out their findings to explain how different impacts may arise, many of the studies failed to report adequately their methods of data collection and analysis.

There is a potential in any studies looking at the quantitative impact of protected areas that respondents will bias their responses in an effort to influence protected area governance. Some questionnaire-based studies attempted to minimise this strategic bias by clearly stating that interview and questionnaire results would be used solely for research purposes, other studies did not acknowledge this potential bias or attempt to reduce it. Of the 305 outcome measures extracted from 79 included studies, 92 outcome measures (30\%) involved 'reported changes'. For these data the comparator is implicit in the respondent's reply; they are reporting a change over time due to the protected area. Whilst these results are relevant (externally valid) to the 
review in hand, they can be susceptible to significant recall bias and questioning bias (elements of internal validity). Several studies attempted to retrospectively elicit opinions and attitudes towards protected area establishment many years after the event, which is similarly open to substantial sources of bias.

For these reasons, results in the form of reported changes which involve significant recall should be viewed with caution, and studies critiqued in depth for potential sources of bias. We attempted to account for these sources of bias during critical appraisal using our 'susceptibility to bias' scoring system.

Sixty-six of the 79 studies accepted following critical appraisal collected data in the form of self-reported measures. Fifty-six of these articles failed to provide details of the questionnaires given to respondents, and only two articles provided a copy of the survey instrument in full $[76,105]$. Without details of the questioning involved in these surveys it is difficult to assess questioning bias.

Variation in all the question elements (PECO; population, exposure, comparator, outcome) and the high degree of specificity in outcome measures identified in this review provides problems for synthesis. In particular, studies based on self-reported measures commonly asked very specific questions that could not then be synthesised along with other similar outcomes. Similarly, a high degree of variability in the choice and design of comparators prevented synthesis. In some studies, the inside-outside comparison was open to many confounding factors that cloud the link between protected area presence and impacts. This highlights the difficulty of balancing minimisation of spillover effects, whereby the comparator population is close enough to feel the effects of the exposure, and control of non-target variables. Whilst some studies accounted for this problem by including confounding variables in statistical models, many others did not. Furthermore, very few studies examined differences in environmental conditions between the comparator and exposure populations.

High susceptibility to bias in most studies limits ability to attribute outcomes/impacts to presence of PAs. Fortyfive studies were excluded during critical appraisal due to flaws in experimental design and data analysis, or due to a lack of methodological detail. However, many studies included after the first stage of critical appraisal also failed to account for confounding variables, selected replicates in a non-random manner, and used opportunistic methodology. The most frequently occurring factor that affected the susceptibility to bias score in included studies, however, was a failure to appropriately report their methodology. Significant details such as recall period, response rate, item pool balance and order, sample selection process, sample size, and sample location were not disclosed in a large number of cases. Together, these factors limit the ability to attribute the reported impacts to protected areas.
There is a lack of primary studies estimating impact of PAs on human well-being using direct measurement techniques in a BACI format. In addition to a generally high susceptibility to bias, very few studies employed robust comparators over appropriate time frames in order to maximise evidence linking protected areas to observed human well-being impacts. Only one included study used a full BACI design to account for spatial and temporal confounding variables. Only three studies used direct data collection.

We found a surprisingly small number of studies on health of populations. Only nine studies reported data on human health impacts of protected areas. This is surprising since the majority of articles in this review purport to measure human well-being. Difficulties in ethical approval for human study may account in part for the paucity of health studies.

\section{Review conclusions}

\section{Implication for policy/management}

The evidence base provides a range of possibilities to inform but little evidence to support decision making on how to maximise positive impacts of PAs on human wellbeing. The diversity of studies and of outcomes measured, together with the diversity (or lack of clear signal) in the data suggests that impacts of PAs are highly context dependent. However, the evidence base is insufficient to provide any power with which to predict impacts on wellbeing from a knowledge of their context. It logically follows that there is an insufficient evidence base to identify circumstances/variables/effect modifiers that might lead to greater or lesser impact. At present, the available evidence base is failing to inform policy on the progress (or lack of it) being made, since 1992, toward lessening negative and promoting positive impact of PAs on human well-being.

\section{Implication for research}

The nature of the research reported to date forms a diverse and fragmented evidence base that is insufficiently developed to reliably inform future policy decisions (recognising that many included studies did not set out to address the review question). Many studies appear to have been conducted opportunistically and lack baseline measures. There is no evidence of a strategic approach or strategic investment to this field of research beyond individual research group initiatives. If a sufficient evidence base is to be formed then there is a need for concerted programme of research rather than an uncoordinated short term opportunistic approach.

The diversity of outcome measures and the consequent difficulty for synthesis suggests a need for use of standard indicators of human well-being that allow comparison among studies and meaningful synthesis of evidence. 
Comparative research needs to progress from $\mathrm{PA} /$ no PA to PA type A/PA type B comparisons. Comparisons should be made between potential proximate causes of positive or negative impacts when the ultimate cause is PA establishment/management. This review suggests some of the candidates to be governance models, existing social capital, cultural diversity and poverty index.

It would be helpful to research efforts for funders to find consensus on minimum standards for methodologies, for both qualitative and quantitative evidence, that provide improved quality and thus reliability of data. The large proportion of included studies that suffered high susceptibility to bias is an indicator of such a need and also an indicator that scarce research resources are not being used effectively.

\section{Recommended study design}

In order to better assess the impacts of protected areas on human well-being we make the following recommendations for future research study design and reporting;

Methodological detail; Studies must report sufficient
details regarding the location of sample sites (in
relation to the protected area boundaries in particular),
the degree of replication, the data collection tool
(e.g. quote questions posed to respondents in
questionnaires), the method of sample selection (e.g.
random or purposeful), and the times and duration of
sampling. This is not an exhaustive list, and sufficient
detail must be provided to allow the sampling to be
repeated. Where information cannot fit within
published articles these details should be provided in
supplementary material.
Baseline assessment; Where changes following
establishment or change in protected area governance
are being investigated, adequate baselines must be
assessed. Although this is difficult and requires
planning prior to the intervention, full 'before-after-
control-intervention' (BACI) study design is vital to
account for confounding temporal and spatial
confounding factors. By assessing baselines, any
differences between intervention and comparator
populations can be compared relative to the starting
conditions to strengthen the evidence towards causation.

\section{Matched controls}

'Control' or 'comparator' populations are vital to enable conclusions to be drawn about impacts in the absence of the intervention. A reliable comparison requires that as many other variables describing the environment are held constant or matched between comparator and intervention populations, allowing only the intervention to change in an ideal situation. In practice this is very difficult (and why baseline assessment is important), and there is often a payoff between maximising similarity and minimising spillover (the overflow of impacts from the intervention into the nearby comparator). Statistical tests can help to confirm similarity across intervention and comparator populations, and descriptive variables can be included in models that test for the significance of the intervention in order to account for differences that might occur.

\section{Replication}

Care must be taken to ensure that there is an appropriate trade-off between a study's accuracy and its precision. When combining many studies in a synthesis, more accurate results are preferable to more precise ones. For example, a study that measures daily resource extraction over a year in ten households from one intervention and one comparator village is less likely to reflect the true impact of the intervention than a study that measures daily resource extraction over a month from 12 intervention and 12 comparator villages. This spectrum is not clear-cut, however, and the allocation of resources to pseudoreplication (improving precision) and true replication (improving accuracy) must be considered carefully. Indeed, the scale at which conclusions will be drawn defines what is pseudoreplication and what is true replication, and this definition may be different for the author and the systematic reviewer.

\section{Statistics}

Statistics, both in summarising results and analysing patterns, must be used with great care. We recommend that a statistician be consulted during experimental design in order to optimise design for analysis. The use of models that account for changes in non-target variables across temporal and spatial scales are recommended, but tests for differences in confounders between intervention and comparator populations are also appropriate. Where information can be presented in summary statistics (e.g. mean/median and standard deviation/confidence intervals) this will aid future meta-analysis.

\section{Additional files}

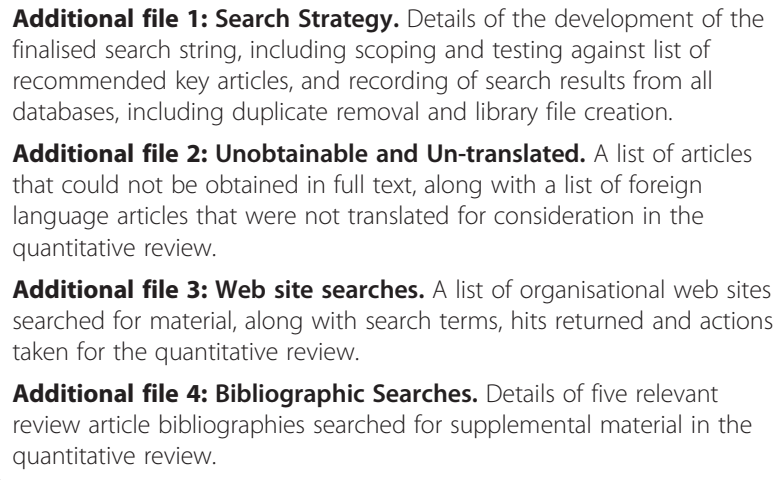

Additional file 3: Web site searches. A list of organisational web sites searched for material, along with search terms, hits returned and actions taken for the quantitative review.

Additional file 4: Bibliographic Searches. Details of five relevant review article bibliographies searched for supplemental material in the quantitative review. 
Additional file 5: Coding Tool. The coding tool used to assess articles at full text for the qualitative review, including the coding of outcomes used in both reviews.

Additional file 6: Excluded. Lists of articles excluded at full text assessment from both the qualitative and quantitative reviews along with reasons.

Additional file 7: Narrative Synthesis. Full narrative synthesis tables for 79 included studies in quantitative review.

Additional file 8: Supplementary Descriptive Statistics for

'Qualitative Synthesis'. Descriptive statistics for the 305 articles included in the systematic map of the first stage of analysis of the qualitative review.

Additional file 9: Qualitative Synthesis. The full synthesis of 30 studies considered in the qualitative review, from which the summary synthesis was produced.

Additional file 10: Table S1. Summary table of included studies and their measured outcomes in the quantitative review that were scored as having 'low' or 'medium' susceptibility to bias (Susc. to Bias).

Additional file 11: Details of the information considered during critical appraisal from each of the 79 studies included in the quantitative review.

\section{Competing interest}

There are no potential conflicts of interest to report.

\section{Authors' contributions}

ASP managed and planned the conduct of the SR. SO managed the conduct of the qualitative section. SD and NRH undertook searches. Quantitative Review; SD and NRH screened for inclusion. NRH carried out data extraction and critical appraisal. JPGJ, JRH, NH and TK assisted in planning, interpretation and synthesis. Qualitative Review; MB, KD, HH SO and CV undertook screening, data extraction and synthesis. All authors contributed to writing and approved the final manuscript.

\section{Acknowledgments}

We thank Sandra Diaz and Tom Hammond for their help in administering the progress of this review.

\section{Sources of support}

This review was funded by the Scientific and Technical Advisory Panel of the Global Environment Facility.

\section{Author details}

'Centre for Evidence-Based Conservation, School of Environment, Natural Resources and Geography, Bangor University, LL57 2UW Bangor, Gwynedd, UK. ${ }^{2}$ Evidence-informed Policy and Practice Information and Coordinating Centre (EPPI-Centre), Institute of Education, University of London, 20 Bedford Way, WC1H ONR London, UK. ${ }^{3}$ School of Environment, Natural Resources and Geography, Bangor University, LL57 2UW Bangor, Gwynedd, UK

Received: 9 January 2013 Accepted: 17 July 2013

Published: 28 October 2013

\section{References}

1. Jenkins CN, Joppa L: Expansion of the global terrestrial protected area system. Biol Conserv 2009, 142:2166-2174.

2. Bertzky B, Corrigan C, Kemsey J, Kenney S, Ravilious CCB, Burgess N: Protected Planet Report 2012: Tracking progress towards global targets for protected areas. Cambridge, UK: IUCN, Gland, Switzerland and UNEP-WCMC; 2012.

3. Brockington D, Igoe J, SCHMIDT-SOLTAU K: Conservation, human rights, and poverty reduction. Conserv Biol 2006, 20:250-252.

4. Dowie M: Conservation refugees: The hundred-year conflict between global conservation and native peoples. Cambridge USA: MIT Press; 2009.

5. Turner WR, Brandon K, Brooks TM, Gascon C, Gibbs HK, Lawrence KS, Mittermeier RA, Selig ER: Global biodiversity conservation and the alleviation of poverty. BioScience 2012, 62:85-92.

6. Kramer RA, Sharma NP, Munasinghe M: Valuing tropical forests: methodology and case study of Madagascar. Madagascar: World Bank Publications; 1995.

7. Adams C, Seroa da Motta R, Ortiz RA, Reid J, Ebersbach Aznar C, de Almeida Sinisgalli PA: The use of contingent valuation for evaluating protected areas in the developing world: Economic valuation of Morro do Diabo State Park, Atlantic Rainforest, São Paulo State (Brazil). Ecol Econ 2008, 66:359-370

8. Ferraro PJ, Hanauer MM, Sims KRE: Conditions associated with protected area success in conservation and poverty reduction. Proc Natl Acad Sci 2011, 108:13913-13918.

9. West P, Igoe J, Brockington D: Parks and peoples: the social impact of protected areas. Annual Reviews in Anthropology 2006, 35:251-277.

10. Sutherland WJ, Adams WM, Aronson RB, Aveling R, Blackburn TM, Broad S, Ceballos G, Cote IM, Cowling RM, Da Fonseca GAB, et al: One hundred questions of importance to the conservation of global biological diversity. Conserv Biol 2009, 23:557-567.

11. Adams WM, Aveling R, Brockington D, Dickson B, Elliott J, Hutton J, Roe D, Vira B, Wolmer W: Biodiversity conservation and the eradication of poverty. Science 2004, 306:1146-1149.

12. Dudley N: Guidelines for Applying Protected Area Managment Categories. Gland Switzerland: World Conservation Union; 2009.

13. Pullin AS, Bangpan M, Dalrymple S, Dickson K, Healey JR, Hockley N, Jones JPG, Knight TM, Oliver S: Human well-being impacts of terrestrial protected areas? CEE protocol 11-009 Collaboration for Environmental Evidence: wwwenvironmentalevidenceorg/SR11009html; 2012.

14. Neuman L: Social Research Methods: Qualitative and Quantitative Approaches. New York: Allyn and Bacon; 1997.

15. Gough D, Thomas J, Oliver S: Clarifying differences between review designs and methods. Systematic Reviews 2012, 1:28.

16. Oliver SR, Rees RW, Clarke-Jones L, Milne R, Oakley AR, Gabbay J, Stein K, Buchanan P, Gyte G: A multidimensional conceptual framework for analysing public involvement in health services research. Health Expect 2008, 11:72-84.

17. Ritchie J, Spencer L: Qualitative data analysis for applied policy research. In Analyzing Qualitative Data. Edited by Bryman A, Burgess RG. London: Routledge; 1994:305-329.

18. Pope C, Ziebland S, Mays N: Qualitative research in health care: Analysing qualitative data. BMJ: British Medical Journal 2000, 320:114.

19. Thomas J, Harden A, Newman M: Synthesis: combining results systematically and appropriately. In Introducing systematic reviews. Edited by Gough D, Oliver S, J. T. London: Sage Publications; 2012.

20. Vasak K: Human Rights: A Thirty-Year Struggle: the Sustained Efforts to give Force of law to the Universal Declaration of Human Rights. UNESCO Courier 30:11. Paris: United Nations Educational, Scientific, and Cultural Organization; 1977.

21. Borenstein M, Hedges LV, Higgins JPT, Rothstein HR: Introduction to metaanalysis. Chichester, UK: Wiley; 2011.

22. Strickland-Munro J, Moore S: Indigenous involvement and benefits from tourism in protected areas: a study of Purnululu National Park and Warmun Community, Australia. J Sustain Tour 2013, 21:26-41.

23. Allendorf TD, Smith JLD, Anderson DH: Residents' perceptions of Royal Bardia National Park, Nepal. Landscape and Urban Planning 2007, 82:33-40.

24. Haukeland JV: Tourism stakeholders' perceptions of national park management in Norway. J Sustain Tour 2011, 19:133-153.

25. Castillo A, Magana A, Pujadas A, Martínez L, Godínez C: Understanding the interaction of rural people with ecosystems: A case study in a tropical dry forest of Mexico. Ecosystems 2005, 8:630-643.

26. Mehring M, Seeberg-Elverfeldt C, Koch S, Barkmann J, Schwarze S, StollKleemann S: Local institutions: Regulation and valuation of forest useEvidence from Central Sulawesi, Indonesia. Land Use Policy 2011, 28:736-747.

27. Petrzelka P, Marquart-Pyatt S: "With the Stroke of a Pen": Designation of the Grand Staircase Escalante National Monument and the Impact on Trust. Hum Ecol 2013, 41:285-297.

28. Gerritsen PRW: Diversity at stake: a farmers' perspective on biodiversity and conservation in western Mexico. Mexico: Wageningen Universiteit Holanda; 2002.

29. Diaw MC, Tiani AM: Fences in our heads: A discourse analysis of the Korup resettlement stalemate. J Sustain For 2010, 29:221-251.

30. Nguiffo S: One forest and two dreams: The constraints imposed on the Baka in Miatta by the Dja Wildlife Reserve. In Indigenous people and protected areas in Africa. Edited by Nelson J, Hossack L, Moreton-in-Marsh. UK: Forest Peoples Programme; 2003:195-214

31. Sletten $\mathrm{M}$, Vedeld P, Kaboggoza J: To co-operate or not to co-operate? a study of collaborative management planning in Mount Elgon National Park. Uganda: NORAGRIC working paper; 2008.

32. Bolaane M: The impact of game reserve policy on the River BaSarwa/ Bushmen of Botswana. Social Policy \& Administration 2004, 38:399-417. 
33. Milgroom J, Spierenburg M: Induced volition: Resettlement from the Limpopo National Park, Mozambique. J Contemp Afr Stud 2008, 26:435-448.

34. Mbaiwa JE: Wildlife resource utilisation at Moremi Game Reserve and Khwai community area in the Okavango Delta, Botswana. $J$ Environ Manage 2005, 77:144-156.

35. Torri MC: Conservation, relocation and the social consequences of conservation policies in protected areas: Case study of the Sariska Tiger Reserve. India. Conservation and Society 2011, 9:54.

36. Keskitalo ECH, Lundmark L: The controversy over protected areas and forest-sector employment in Norrbotten, Sweden: forest stakeholder perceptions and statistics. Soc Nat Resour 2009, 23:146-164.

37. Spenceley A, Goodwin H: Nature-based tourism and poverty alleviation: impacts of private sector and parastatal enterprises in and around Kruger National Park, South Africa. Current Issues in Tourism 2007 10:255-277.

38. Songorwa AN: Community-based wildlife management (CWM) in Tanzania: Are the communities interested? World Dev 1999, 27:2061-2079.

39. Stone M, Wall G: Ecotourism and community development: case studies from Hainan, China. Environ Manage 2004, 33:12-24.

40. Bruyere BL, Beh AW, Lelengula G: Differences in perceptions of communication, tourism benefits, and management issues in a protected area of rural Kenya. Environ Manage 2009, 43:49-59.

41. Slater R: Between a rock and a hard place: contested livelihoods in Qwaqwa National Park, South Africa. Geogr J 2002, 168:116-129.

42. Bizikova L, Nijnik M, Kluvanková-Oravská T: Sustaining multifunctional forestry through the developing of social capital and promoting participation: a case of multiethnic mountain communities. Small-Scale Forestry 2012, 11:301-319.

43. Ormsby A, Kaplin BA: A framework for understanding community resident perceptions of Masoala National Park, Madagascar. Environ Conserv 2005, 32:156-164.

44. First Peoples Worldwide: Okiciyab: To help each other-Promoting best practices in Indigenous community Development. 2006. Final report, June.

45. ESCO: Biosphere reserves: The Seville Strategy and the Statutory Framework of the World Network. Paris: UNESCO; 1996.

46. World Parks Congress: The Durban Action Plan. Durban, South Africa: World Parks Congress; 2003.

47. Herrold-Menzies M: From Adversary to Partner: The Evolving Role of Caohai Nature Reserve in the Lives of Reserve Residents. Canadian Journal of Development Studies/Revue canadienne d'études du développement 2006, 27:39-50.

48. Lunstrum E: Mozambique, neoliberal land reform, and the Limpopo National Park. Geogr Rev 2008, 98:339-355.

49. Almudi T, Berkes F: Barriers to empowerment: Fighting eviction for conservation in a southern Brazilian protected area. Local Environment 2010, 15:217-232.

50. Davis A: ' Ha! What is the benefit of living next to the park?' Factors limiting in-migration next to Tarangire National Park, Tanzania. Conserv Soc 2011, 9:25-34.

51. Hoole A, Berkes F: Breaking down fences: Recoupling social-ecological systems for biodiversity conservation in Namibia. Geoforum 2010, 41:304-317.

52. Hartter J: Attitudes of rural communities toward wetlands and forest fragments around Kibale National Park, Uganda. Hum Dimens Wildl 2009, 14:433-447.

53. Bedunah DJ, Schmidt SM: Pastoralism and protected area management in Mongolia's Gobi Gurvansaikhan National Park. Dev Chang 2004, 35:167-191.

54. Ogra MV: Human-wildlife conflict and gender in protected area borderlands: a case study of costs, perceptions, and vulnerabilities from Uttarakhand (Uttaranchal), India. Geoforum 2008, 39:1408-1422.

55. Stronza A, Gordillo J: Community views of ecotourism. Ann Tour Res 2008, 35:448-468

56. Yasuda A: The Impacts of Sport Hunting on the Livelihoods of Local People: A Case Study of Bénoué National Park, Cameroon. Soc Nat Resour 2011, 24:860-869.

57. Nyahongo J, Holmern T, Kaltenborn BP, Roskaft E: Spatial and temporal variation in meat and fish consumption among people in the western Serengeti, Tanzania: the importance of migratory herbivores. Oryx 2009, 43:258.

58. Sarker A, Røskaft E: Human attitudes towards the conservation of protected areas: a case study from four protected areas in Bangladesh. Oryx 2011, 45:391-400
59. Bajracharya SB, Furley PA, Newton AC: Impacts of community-based conservation on local communities in the Annapurna Conservation Area, Nepal. Biodivers Conserv 2006, 15:2765-2786.

60. Mehta JN, Kellert SR: Local attitudes toward community-based conservation policy and programmes in Nepal: a case study in the Makalu-Barun Conservation Area. Environ Conserv 1998, 25:320-333.

61. Naughton-Treves L, Alix-Garcia J, Chapman CA: Lessons about parks and poverty from a decade of forest loss and economic growth around Kibale National Park, Uganda. Proc Natl Acad Sci 2011, 108:13919-13924.

62. Rinzin C, Vermeulen WJV, Wassen MJ, Glasbergen P: Nature Conservation and Human Well-Being in Bhutan An Assessment of Local Community Perceptions. J Environ Dev 2009, 18:177-202.

63. Okello MM, Buthmann E, Mapinu B, Kahi HC: Community opinions on wildlife, resource use and livelihood competition in Kimana Group Ranch near Amboseli, Kenya. Open Conservation Biology Journal 2011, 5:1-12.

64. Lundgren T: Environmental protection and impact on adjacent economies: evidence from the Swedish mountain region. Growth and Change 2009, 40:513-532.

65. Richardson RB, Fernandez A, Tschirley D, Tembo G: Wildlife Conservation in Zambia: impacts on rural household welfare. World Dev 2012, 40:1068-1081.

66. Sims KRE: Conservation and development: Evidence from Thai protected areas. J Environ Econ Manag 2010, 60:94-114.

67. Andam KS, Ferraro PJ, Sims KR, Healy A, Holland MB: Protected areas reduced poverty in Costa Rica and Thailand. Proc Natl Acad Sci 2010, 107:9996-10001.

68. Canavire-Bacarreza G, Hanauer MM: Estimating the impacts of bolivia's protected areas on poverty. World Dev 2012, 41:265-285.

69. Ferraro PJ, Hanauer MM: Protecting Ecosystems and Alleviating Poverty with Parks and Reserves: 'Win-Win'or Tradeoffs? Environ Resour Econ 2011 48:269-286.

70. Foerster S, Wilkie DS, Morelli GA, Demmer J, Starkey M, Telfer P, Steil M: Human livelihoods and protected areas in Gabon: a cross-sectional comparison of welfare and consumption patterns. Oryx 2011, 45:347-356.

71. Cardozo M: Economic displacement and local attitude towards protected area establishment in the Peruvian Amazon. Geoforum 2011, 42:603-614.

72. Kayser D, Sobrevila C, Ledec G: From Planning to the Implementation of a Successful Conservation and Socio-Economic Model. South Africa: Report from The World Bank and Addo Elephant National Park; 2011.

73. Saayman M, Saayman A: Regional development and national parks in South Africa: Lessons learned. Tour Econ 2010, 16:1037-1064.

74. Ninan KN: Non Timber Forest Products and Biodiversity Conservation: a Study of Tribals in a Protected Area in India. In Conserving and Valuing Ecosystem Services and Biodiversity: Economic, Institutional and Social Challenges. Edited by Ninan KN. London: Earthscan; 2012:99-112.

75. ETFRN NEWS 39/40: Globalisation, localisation and tropical forest management. http://www.etfrn.org/etfrn/newsletter/pdf/etfrnnews3940.pdf

76. Phamtrong N, Swan S: An investigation into the drivers of forest encroachment in Bidoup Nui Ba National Park, Vietnam. Vietnam: Report to WWF Greater Mekong - Vietnam Country Programme; 2009

77. Korhonen K, Rahkonen O, Hemminki E: Implications of integrated nature conservation for human reproductive health: a case study from Ranomafana National Park, Madagascar. Dev South Afr 2004, 21:603-621.

78. Mishra C: Socioeconomic transition and wildlife conservation in the Indian Trans-Himalaya. Journal of the Bombay Natural History Society 2000, 97:25-32

79. Sheppard DJ, Moehrenschlager A, McPherson JM, Mason JJ, Pattenden J, Khandker S, Koolwal G, Biggs B, King L, Basu S: Ten years of adaptive community-governed conservation: evaluating biodiversity protection and poverty alleviation in a West African hippopotamus reserve. Environ Conserv 2010, 37:270-282.

80. Shrestha RK, Alavalapati JRR: Linking conservation and development: An analysis of local people's attitude towards Koshi Tappu wildlife reserve, Nepal. Environ Dev Sustain 2006, 8:69-84.

81. Bonaiuto $\mathrm{M}$, Carrus $\mathrm{G}$, Martorella $\mathrm{H}$, Bonnes $\mathrm{M}$ : Local identity processes and environmental attitudes in land use changes: The case of natural protected areas. J Econ Psychol 2002, 23:631-653.

82. Jim C, Xu SSW: Stifled stakeholders and subdued participation: interpreting local responses toward Shimentai Nature Reserve in South China. Environ Manage 2002, 30:327-341.

83. Gubbi S, Linkie M, Leader-Williams N: Evaluating the legacy of an integrated conservation and development project around a tiger reserve in India. Environ Conserv 2008, 35:331 
84. Infield M, Namara A: Community attitudes and behaviour towards conservation: an assessment of a community conservation programme around Lake Mburo National Park, Uganda. Oryx 2001, 35:48-60.

85. Sekhar NU: Crop and livestock depredation caused by wild animals in protected areas: the case of Sariska Tiger Reserve, Rajasthan, India. Environ Conserv 1998, 25:160-171.

86. Fiallo EA, Jacobson SK: Local Communities and Protected Areas: Attitudes of Rural Residents Towards Conservation and Machalilla National Park, Ecuador. Environ Conserv 1995, 22:241-249.

87. Harada K: Attitudes of local people towards conservation and Gunung Halimun National Park in West Java, Indonesia. J For Res 2003, 8:271-282.

88. Hartter J, Goldman AC: Life on the Edge: Balancing Biodiversity, Conservation, and Sustaining Rural Livelihoods around Kibale National Park, Uganda. Focus Geogr 2009, 52:11-17.

89. Ite DUE: Community perceptions of the Cross River national park Nigeria. Environ Conserv 1996, 23:351-357.

90. Cihar M, Stankova J: Attitudes of stakeholders towards the Podyji/Thaya River Basin National Park in the Czech Republic. J Environ Manage 2006, 81:273-285.

91. Alexander SE: Resident attitudes towards conservation and black howler monkeys in Belize: the Community Baboon Sanctuary. Environ Conserv 2000, 27:341-350

92. Rugendyke B, Son NT: Conservation costs: Nature-based tourism as development at Cue Phuong national park, Vietnam. Asia Pacific Viewpoint 2005, 46:185-200.

93. Saayman M, Saayman A, Ferreira M: The socio-economic impact of the Karoo National Park. Koedoe 2009, 51:1-10.

94. Just RE, Hueth DL, Schmitz A: The Welfare Economics of Public Policy: A Practical Approach to Project and Policy Evaluation. Cheltenham, UK: Edward Elgar; 2004.

95. Shrestha RK, Alavalapati JRR, Seidl AF, Weber KE, Suselo TB: Estimating the local cost of protecting Koshi Tappu Wildlife Reserve, Nepal: a contingent valuation approach. Environ Dev Sustain 2007, 9:413-426.

96. Jemal H, Bogale A, Hagedorn $\mathrm{K}$ : Welfare loss for pastoralists due to wildlife protection areas: the case of Awash National Park, Ethiopia. Quarterly Journal of International Agriculture 2008, 47:217-238.

97. Kremen C, Niles J-O, Dalton MG, Daily GC, Ehrlich PR, Fay JP, Grewal D, Guillery RP: Economic incentives for rainforest conservation across scales. Science 2000, 288:1828-1832.

98. Han $F$, Yang Z, Wang $H, X u X$ : Estimating willingness to pay for environment conservation: a contingent valuation study of Kanas Nature Reserve, Xinjiang, China. Environ Monit Assess 2011, 180:451-459.

99. Maharana I, Rai S, Sharma E: Environmental economics of the Khangchendzonga National Park in the Sikkim Himalaya, India. GeoJournal 2000, 50:329-337.

100. Jones N, losifides T, Evangelinos KI, Florokapi I, Dimitrakopoulos PG: Investigating knowledge and perceptions of citizens of the National Park of Eastern Macedonia and Thrace, Greece. International Journal of Sustainable Development \& World Ecology 2012, 19:25-33.

101. Asciuto A, Schimmenti E: Comparison between Willingness-to-Pay expressed by a panel of forestry experts and by a sample of non-expert respondents in a pilot survey conducted for a contingent valuation study. Mediterranean Journal of Economics, Agriculture and Environment 2005, 4:42-51.

102. Abbot JIO, Mace R: Managing protected woodlands: fuelwood collection and law enforcement in Lake Malawi National Park. Conserv Biol 2001, 13:418-421.

103. Kavanagh J, Oliver S, Lorenc T, Caird J, Tucker H, Harden A, Greaves A Thomas J, Oakley A: School-based cognitive-behavioural interventions: A systematic review of effects and inequalities. Heal Sociol Rev 2009, 18:61-78.

104. Petticrew M, Tugwell P, Kristjansson E, Oliver S, Ueffing E, Welch V: Damned if you do, damned if you don't: subgroup analysis and equity. J Epidemiol Community Health 2012, 66:95-98.

105. Reid $\mathrm{R}$, Stone M, Whitely $\mathrm{T}$ : Economic value of wilderness protection and recreation in British Columbia. (Victoria BC): FRDA Working Paper; 1995.

doi:10.1186/2047-2382-2-19

Cite this article as: Pullin et al:: Human well-being impacts of terrestrial protected areas. Environmental Evidence 2013 2:19.

\section{Submit your next manuscript to BioMed Central and take full advantage of:}

- Convenient online submission

- Thorough peer review

- No space constraints or color figure charges

- Immediate publication on acceptance

- Inclusion in PubMed, CAS, Scopus and Google Scholar

- Research which is freely available for redistribution 